\title{
2 Fixation and effective size in a haploid-diploid population with asexual reproduction
}

4 *Kazuhiro Bessho, **Sarah P. Otto

5 * Saitama Medical University, 38 Morohongo Moroyama-machi, Iruma-gun, Saitama 350-0495,

6 Japan

7 **Department of Zoology, The University of British Columbia, Vancouver, BC, V6T 1Z4,

\section{Canada}

9 * besshokazuhiro.reserch@gmail.com; (+81) 049-276-2030

$10 * *$ otto@zoology.ubc.ca; (+1) 604-822-2778

$12 *$ Corresponding author

13 besshokazuhiro.reserch@gmail.com (KB)

$14 *$ Co-corresponding author

15 otto@zoology.ubc.ca (SPO)

17 Keywords: haploid-diploid life cycle, Wright-Fisher model, fixation probability, variance

18 effective population size, class reproductive value 


\section{Abstract}

26 The majority of population genetic theory assumes fully haploid or diploid organisms

27 with obligate sexuality, despite complex life cycles with alternating generations being commonly

28 observed. To reveal how natural selection and genetic drift shape the evolution of haploid-diploid

29 populations, we analyze a stochastic genetic model for populations that consist of a mixture of

30 haploid and diploid individuals, allowing for asexual reproduction and niche separation between

31 haploid and diploid stages. Applying a diffusion approximation, we derive the fixation

32 probability and describe its dependence on the reproductive values of haploid and diploid stages,

33 which depend strongly on the extent of asexual reproduction in each phase and on the ecological

34 differences between them. 


\section{1. Introduction}

Sexual reproduction in eukaryotes generally consists of an alternation of generations,

38 where meiosis halves the number of chromosomes to produce haploids and syngamy brings

39 together haploid gametes to produce diploids. The extent of development in each ploidy phase

40 varies substantially (Bell 1982; 1994). In diplontic organisms, at one extreme, development and

41 growth occur only in the diploid phase, as is observed in most animals. Haplontic organisms, at

42 the other extreme, undergo mitotic growth only in the haploid stage, as is seen in some green

43 algae. In between these extremes, many terrestrial plants, macroalgae, and fungi exhibit both

44 haploid and diploid growth (haploid-diploid life cycles). These stages are typically free living in

45 macroalgae, with either macroscopically similar (isomorphic) or distinct (heteromorphic) forms

46 in the haploid and diploid stage (Raper and Flexer 1970; Wilson 1981; Mable and Otto 1998;

47 Coelho 2007).

48 To explain variation in life cycles, several theoretical models have analyzed the

49 deterministic dynamics of a modifier allele that alters the time spent in haploid and diploid

50 phases (e.g., Perrot et al. 1991; Otto and Goldstein 1992; Goldstein 1992; Otto 1994; Orr and

51 Otto 1994; Jenkins and Kirkpatrick 1995; Otto and Marks 1996; Scott and Rescan 2017).

52 However, there are some gaps between these models and the complexities seen in many

53 haploid-diploid species. For example, these models often treat haploid and diploid individuals as

54 ecologically equivalent, despite the frequent observation of niche differences and seasonal shifts

55 in prevalence (e.g., Slocum 1980; Dethier 1981). Most of these models also assume obligate

56 sexuality (but see Otto and Marks 1996), despite asexuality being frequently observed among

57 haploid-diploid species ("asexual looping”). Furthermore, while several models have explored

58 how haploid-diploid life cycles might evolve, the impact of haploid-diploid life cycles on 
59 evolutionary processes remains underexplored (see, e.g., Bessho and Otto 2017 on the impact on

60 fixation probabilities and Immler et al. 2012 on the maintenance of variation).

61 Here we contribute to evolutionary theory for haploid-diploid populations by

62 calculating the fixation probability of mutations using a stochastic genetic model. This builds

63 upon our previous work (Bessho and Otto 2017) by accounting for asexual looping and niche

64 differences between ploidy phases, both of which are common in macroalgae (Bell 1982; de

65 Wreede and Klinger 1988; Hawkes 1990). Haploid and diploid phases often differ

66 physiologically, and even isomorphic haploids and diploids may differ ecologically (Hannach

67 and Santelices 1985; Destombe et al. 1993; Dyck and de Wreede 2006; Thornber et al., 2006;

68 Vieira et al. 2018). We therefore explore different forms of density dependence, acting either

69 globally on the total population size (as in Bessho and Otto 2017) or locally on the population

70 size of haploids and diploids separately (Figure 1). We show that the fate of a mutation depends

71 strongly on the reproductive values of haploids and diploids, which in turn depend on the extent

72 of asexual reproduction and ecological differences between the phases.

\section{2. Model}

75 In Bessho and Otto (2017), we calculated the fixation probabilities by tracking the

76 dynamics of a resident allele $(R)$ and a mutant allele $(M)$ in haploid and diploid individuals, using

77 both a Wright-Fisher and a Moran model. In that model, reproduction was obligately sexual,

78 individuals were ecologically equivalent, and the total population size was held constant (global

79 density dependence). Below, we calculate the fixation probability by first considering asexual

80 reproduction in each phase, assuming that haploids and diploids are ecologically equivalent

81 (global population regulation), and then determine how these results are affected by niche

82 differences (local population regulation that is ploidy specific). 
84 2.1. Haploid-diploid Wright-Fisher model with global regulation and asexual looping

85 Let $X_{(G T)}(t)$ be a random variable that represents the number of individuals with

86 "genotype" $(G T)$ at time $t$ with resident $(R)$ and mutant alleles $(M)$, and let $x_{(G T)}(t)$ represent a

87 particular outcome of this random variable. In the global regulation model, we assume a constant

88 population, $x_{R}+x_{M}+x_{R R}+x_{R M}+x_{M M}=N_{t o t}$, that is strictly regulated regardless of the

89 ploidy of the individuals.

90 The reproductive output and the degree of asexuality are characterized by $w_{(G T)}$ and

$91 a_{H}$ for haploids $[(G T)=R$ or $M]$ and $w_{(G T)}$ and $a_{D}$ for diploids $[(G T)=R R, R M$, and $M M]$.

92 Specifically, diploid individuals produce $\left(1-a_{D}\right) w_{(G T)}$ haploid spores (sexual reproduction)

93 and $a_{D} w_{(G T)}$ diploid offspring (asexual loop). Similarly, haploids produce $\left(1-a_{H}\right) w_{(G T)} / 2$

94 female gametes (sexual reproduction) and $a_{H} w_{(G T)}$ haploid offspring (asexual loop), where we

95 assume that the species is monoecious and invests equal resources in male and female gametes.

96 During syngamy, we assume that male gametes are not limiting, that mating is random, and that

97 female gametes are successfully fertilized with male gametes, at a rate $f_{(G T)}[(G T)=R$ or $M]$,

98 becoming diploid zygotes. For clarity, we describe the model with non-overlapping generations,

99 although we note that overlapping generations can be considered by including surviving adults in

100 the counts of asexual offspring $\left(a_{D} w_{(G T)}\right.$ and $\left.a_{H} w_{(G T)}\right)$.

101 We define the selection coefficient $\left(s_{(G T)}\right)$ and the degree of dominance $(h)$ acting upon

102 the mutant allele such that: $f_{M}=f_{R}\left(1-s_{M}^{f}\right), w_{M}=w_{R}\left(1-s_{M}^{w}\right), w_{R M}=w_{R R}\left(1-s_{R M}^{w}\right)$,

$103 w_{M M}=w_{R R}\left(1-s_{M M}^{w}\right)$, and $h=s_{R M}^{w} / s_{M M}^{w}$. To perform the diffusion approximation, we assume

104 that selection is weak, $s_{M}^{f}=\epsilon \tilde{S}_{M}^{f}$ and $s_{(G T)}^{w}=\epsilon \tilde{S}_{(G T)}^{w}$, where $\epsilon$ is a small parameter. 
108 populations separately, which may occur if they have different resource needs or utilize different

109 habitats or microhabitats (for short-hand, we refer to this case as "local regulation"). More

110 specifically, we assume that the population size of haploids and diploids is separately regulated

111 and remains constant $N_{H}$ and $N_{D}\left(x_{R}+x_{M}=N_{H}\right.$ and $\left.x_{R R}+x_{R M}+x_{M M}=N_{D}\right)$, respectively.

112 We set $N_{H}+N_{D}=N_{t o t}, \hat{\rho}_{H}^{L}=N_{H} / N_{\text {tot }}$, and $\hat{\rho}_{D}^{L}=N_{D} / N_{\text {tot }}$, which will then allow us to

113 compare the results of local and global regulation. Holding population sizes constant is assumed

114 strictly for mathematical convenience but may be reasonable for populations whose sizes are

115 strongly regulated by the availability of appropriate habitat.

\section{3. Fixation probability in a haploid-diploid population}

118 3.1. Fixation probability in the global regulation model

119 The fixation probability in a haploid-diploid population can be derived using a

120 diffusion approximation (Bessho and Otto 2017), but doing so requires that we approximate the

121 dynamics to reduce the dimensionality from four variables $\left(x_{R}, x_{M}, x_{R R}, x_{R M}, x_{M M}\right.$, which sum to

$122 N_{t o t}$ ) down to one. We do so by using a separation of time scales, deriving the first and second

123 moments of the mutant allele frequency. Specifically, we transform the number of individuals of

124 each genotype, $x_{(G T)}$, into new variables that allow us to separate the slower evolutionary

125 dynamics and the faster ecological dynamics (Appendix A):

$$
\begin{aligned}
& p_{a v e}=c_{H} p_{H}+c_{D} p_{D}, \\
& \delta_{p}=p_{D}-p_{H}, \\
& \eta_{H W}=1-\frac{1}{2 p_{D}\left(1-p_{D}\right)} \frac{x_{R M}}{x_{R R}+x_{R M}+x_{M M}},
\end{aligned}
$$




$$
\rho_{H}=\frac{x_{R}+x_{M}}{N_{t o t}}
$$

126 where $p_{\text {ave }}$ indicates the average allele frequency of haploids and diploids weighted by the class

127 reproductive values $\left(c_{H}\right.$ and $c_{D}$, where $c_{H}+c_{D}=1$, see next paragraph), $\delta_{p}$ indicates the

128 difference in allele frequencies between haploids and diploids, $\eta_{H W}$ indicates the departure from

129 the Hardy-Weinberg equilibrium in diploids, and $\rho_{H}$ indicates the frequency of haploids in the

130 population. Within these equations, the frequencies of mutant alleles in haploids and diploids are

$131 p_{H}=x_{M} /\left(x_{R}+x_{M}\right)$ and $p_{D}=\left(\frac{x_{R M}}{2}+x_{M M}\right) /\left(x_{R R}+x_{R M}+x_{M M}\right)$. As similar variables are

132 used in the model with local population regulation, we use superscripts to indicate the form of

133 population regulation ("Model" is $G$ for global or $L$ for local regulation).

134 The class reproductive values of haploids and diploids are defined as follows. In linear

135 models, "reproductive value" is a measure of the expected fraction of the population in the

136 long-term future that descends from an individual of a particular type (e.g., age or stage class).

137 Class reproductive values, as defined by Taylor (1990) and Rousset (2004, p.153), scale these

138 individual reproductive values up to the whole population of each class (i.e., the product of the

139 individual reproductive values times the class size). In the models considered here, the dynamics

140 are non-linear because of competition for resources $\left(N_{t o t}\right)$. Nevertheless, we can approximate

141 reproductive values by assuming that the population is near equilibrium with only resident alleles

142 and by holding the strength of competition constant (see Supplementary Mathematica file for all

143 calculations). Doing so, we find that the class reproductive values of haploids and diploids,

144 expressed as proportions that sum to one, are:

$$
c_{H}^{G}=\frac{\left(1-a_{H}\right) \frac{f_{R}}{2} w_{R}\left(\hat{\rho}_{H}^{G}\right)^{2}}{\left(1-a_{H}\right) \frac{f_{R}}{2} w_{R}\left(\hat{\rho}_{H}^{G}\right)^{2}+\left(1-a_{D}\right) w_{R R}\left(\hat{\rho}_{D}^{G}\right)^{2}},
$$




$$
c_{D}^{G}=\frac{\left(1-a_{D}\right) w_{R R}\left(\hat{\rho}_{D}^{G}\right)^{2}}{\left(1-a_{H}\right) \frac{f_{R}}{2} w_{R}\left(\hat{\rho}_{H}^{G}\right)^{2}+\left(1-a_{D}\right) w_{R R}\left(\hat{\rho}_{D}^{G}\right)^{2}}
$$

145 where $\hat{\rho}_{H}^{G}=1-\hat{\rho}_{D}^{G}$ is the equilibrium frequency of haploids in the global model (Eq. A.4). As

146 a special case of interest, when populations are purely sexual $\left(a_{H}=a_{D}=0\right)$, we can plug the

147 equilibrium for $\hat{\rho}_{H}^{G}$ from Eq. (A.4) into (2) and show that $c_{H}^{G}=c_{D}^{G}=1 / 2$.

148 As discussed in Appendix A (see also Bessho and Otto 2017), equations (2) provide the

149 only weights that allow ecological and evolutionary time scales to be separated when calculating

150 the average allele frequency in equation (1a), which is why we take that to be the evolutionarily

151 relevant average. Although one might initially think that diploids should count twice as much

152 because they contain two allele copies and that the evolutionarily relevant average allele

153 frequency would depend on the population sizes of haploids and diploids, a strict alternation of

154 generations $\left(a_{H}=a_{D}=0\right)$ ensures that haploids and diploids contribute equally to long-term

155 future generations, so that their reproductive values are equal and the evolutionarily relevant

156 average allele frequency is $p_{\text {ave }}=(1 / 2) p_{H}+(1 / 2) p_{D}$ (Bessho and Otto 2017).

157 As with our previous model, we can track the slow evolutionary dynamics for the

158 expected change in average allele frequency $p_{\text {ave }}$ under weak selection, once the fast ecological

159 dynamics have stabilized, as which point we can show that there are similar allele frequencies in

160 haploids and diploids $\left(\delta_{p} \approx 0\right)$, diploids are approximately at Hardy-Weinberg equilibrium

$161\left(\eta_{H W} \approx 0\right)$, and the ratio of haploids is similar among mutant and resident genotypes $\left(\rho_{H} \approx \hat{\rho}_{H}^{G}\right)$

162 (Appendix A, File S1). Furthermore, to leading order, the second moment of change in allele

163 frequency is equal to the neutral case and can be derived in the diffusion limit $\left(N_{t o t} \rightarrow \infty\right)$.

Given a single variable, $p_{\text {ave }}$, changing slowly over evolutionary time, we can then

165 use standard diffusion methods to calculate the fixation probability of a mutation in a

166 haploid-diploid population, $u\left(p_{0}^{\text {Model }}\right)$, where "Model" is $G$ for global and $L$ for local (considered 
167 in the next section). The diffusion is a function of the first and second moments of change in the

168 mutant allele frequency, $m^{\text {Model }}\left(p_{\text {ave }}\right)$ and $v^{\text {Model }}\left(p_{\text {ave }}\right)$, both measured in time units of $N_{\text {tot }}$

169 generations:

$$
\begin{aligned}
& u\left(p_{0}^{\text {Model }}\right)=\frac{\int_{0}^{p_{0}^{\text {Model }}} \exp \left[-2 Q^{\text {Model }}\left(p^{\prime}\right)\right] d p^{\prime}}{\int_{0}^{1} \exp \left[-2 Q^{\text {Model }}\left(p^{\prime}\right)\right] d p^{\prime}}, \\
& m^{\text {Model }}\left(p_{\text {ave }}\right)=\frac{N_{\text {tot }} p_{\text {ave }}\left(1-p_{\text {ave }}\right)}{2}\left[2 s_{\text {ave }}^{\text {Model }}+2 c_{D}^{\text {Model }} p_{\text {ave }}(1-2 h) s_{\text {MM }}^{W}\right] \\
& v^{\text {Model }}\left(p_{\text {ave }}\right)=\frac{p_{\text {ave }}\left(1-p_{\text {ave }}\right)\left[\left(c_{D}^{\text {Model }}\right)^{2} \widehat{\rho}_{H}^{\text {Model }}+\left(c_{H}^{\text {Model }}\right)^{2}\left(2 \widehat{\rho}_{D}^{\text {Model }}\right)\right]}{\hat{\rho}_{H}^{\text {Model }}\left(2 \widehat{\rho}_{D}^{\text {Model }}\right)} .
\end{aligned}
$$

170 where $p_{0}^{\text {Model }}$ is the initial allele frequency of mutants (we focus on the case with a single initial

171 mutant $\quad \quad \quad p_{0}^{\text {Model }}=1 /\left[N_{\text {tot }}\left(\hat{\rho}_{H}^{\text {Model }}+2 \hat{\rho}_{D}^{\text {Model }}\right)\right] \quad$ and $172 Q^{\text {Model }}(p)=\int\left(m^{\text {Model }}(p) / v^{\text {Model }}(p)\right) d p$. For the global regulation model, the average 173 selection acting upon rare mutant alleles across haploid and diploid stages, $s_{\text {ave }}^{G}$, can be 174 calculated from the first moment equation (Appendix 1) and equals:

$$
\begin{aligned}
& S_{\text {ave }}^{\text {Model }}=c_{H}^{\text {Model }} s_{M}^{w}+c_{D}^{\text {Model }} s_{R M}^{w}+\phi^{\text {Model }} c_{D}^{\text {Model }} \frac{s_{M}^{f}}{2}, \\
& \phi^{\text {Model }}=\frac{\left(1-a_{H}\right) \widetilde{w}_{R} \hat{\rho}_{H}^{\text {Model }}}{\left(1-a_{H}\right) \widetilde{w}_{R} \hat{\rho}_{H}^{\text {Model }}+a_{D} w_{R R} \hat{\rho}_{D}^{\text {Model }} .}
\end{aligned}
$$

175 where $\widetilde{w}_{R}=f_{R} w_{R} / 2$ is the fitness of haploids considering the cost of sex. As we will see later,

176 this equation is valid for local regulation model. The term $\phi^{\text {Model }}$ indicates the fraction of the

177 diploids in the next generation that come from the union of gametes rather than diploid asexual

178 reproduction. With obligately sexual haploid-diploids $\left(a_{H}=a_{D}=0\right.$, where $c_{H}^{\text {Model }}=c_{D}^{\text {Model }}=$

$1791 / 2$ and $\phi^{\text {Model }}=1$ ), these results coincide with those of Bessho and Otto (2017).

181 3.2. Fixation probability in the local regulation model 
183 dependence regulates haploid and diploid populations separately (Figure 1b), by again applying a

184 transformation of variables and separation of time scales. For the local regulation model, the

185 appropriate weights for the average allele frequency are similar to the global regulation model,

186 where now the class reproductive values, expressed as proportions, are:

$$
\begin{gathered}
c_{H}^{L}=\frac{1+\frac{a_{H} w_{R} \hat{\rho}_{H}^{L}}{2+\frac{a_{H} w_{R} \hat{\rho}_{H}^{L}}{\left(1-a_{D}\right) w_{R R} \hat{\rho}_{R} \hat{\rho}_{D}^{L}}+\frac{2}{f_{R}} \frac{a_{D} w_{R R} \hat{\rho}_{D}^{L}}{\left(1-a_{H}\right) w_{R} \hat{\rho}_{H}^{L}}},}{1+\frac{2}{f_{R}} \frac{a_{D} w_{R R} \hat{\rho}_{D}^{L}}{\left(1-a_{H}\right) w_{R} \hat{\rho}_{H}^{L}}} \\
c_{D}^{L}=\frac{a_{D} w_{R R} \hat{\rho}_{D}^{L}}{2+\frac{a_{H} w_{R} \hat{\rho}_{H}^{L}}{\left(1-a_{D}\right) w_{R R} \hat{\rho}_{D}^{L}}+\frac{2}{f_{R}} \frac{\left.a_{H}\right) w_{R} \hat{\rho}_{H}^{L}}{\left(1-a_{1}\right.}} .
\end{gathered}
$$

188 After applying a separation of time scales and conducting a diffusion approximation, we

189 conclude that the solution for the fixation probability in a haploid-diploid population, Eqs. (3),

190 remains valid for the local regulation model (Supplementary Mathematica file), with the average

191 selection coefficient now being given by Eqs. (5).

\subsection{Effective genetic parameters}

Using the first and second moments of change in allele frequency, we derive effective

genetic parameters to compare our results to the dynamics found in the classical model for fully

haploid or fully diploid organisms (Bessho and Otto 2017). More specifically, we define the

effective selection coefficient $\left(s_{e}\right)$, dominance coefficient $\left(h_{e}\right)$, and effective population size $\left(N_{e}\right)$

that would result in the same expected change in allele frequency and variance as in the classical diploid model of selection. 
202 frequency in the same way as Eq. (3b), we can find the effective and dominance selection 203 coefficient from $\Delta p_{\text {ave }} /\left[p_{\text {ave }}\left(1-p_{\text {ave }}\right)\right]=s_{e} h_{e} \quad$ when $\quad p_{\text {ave }}=0 \quad$ and

$204 \Delta p_{\text {ave }} /\left[p_{\text {ave }}\left(1-p_{\text {ave }}\right)\right]=s_{e}\left(1-h_{e}\right)$ when $p_{\text {ave }}=1$, yielding:

$$
\begin{aligned}
& s_{e}^{\text {Model }}=2 s_{\text {ave }}^{\text {Model }}+2 \hat{c}_{D}^{\text {Model }} \frac{(1-2 h) s_{M M}^{w}}{2} . \\
& h_{e}^{\text {Model }}=\frac{2 s_{\text {ave }}^{\text {Model }}}{4 s_{\text {ave }}^{\text {Model }}+2 \hat{c}_{D}^{\text {Model }}(1-2 h) s_{M M}^{w}} .
\end{aligned}
$$

205 When the mutation is additive $(h=1 / 2)$, these effective parameters are $s_{e}^{\text {Model }}=2 s_{\text {ave }}^{\text {Model }}$ and $206 \quad h_{e}^{\text {Model }}=1 / 2$

$$
N_{e}^{\text {Model }}=\frac{p_{\text {ave }}\left(1-p_{\text {ave }}\right)}{2\left(v^{\text {Model }} / N_{\text {tot }}\right)}=\frac{N_{\text {tot }} \hat{\rho}_{H}^{\text {Model }} \hat{\rho}_{D}^{\text {Model }}}{\left(c_{D}^{\text {Model }}\right)^{2} \hat{\rho}_{H}^{\text {Model }}+2\left(c_{H}^{\text {Model }}\right)^{2} \hat{\rho}_{D}^{\text {Model }}} .
$$

212 the classical diploid Wright-Fisher model (Kimura 1957; 1962; Crow and Kimura 1970, p. 427),

213 the fixation probability in a haploid-diploid population given by Eq. 3a can be expressed as:

$$
u\left(p_{0}^{\text {Model }}\right)=\frac{\int_{0}^{p_{0}^{\text {Model }}} \exp \left[-2 N_{e}^{\text {Model }} s_{e}^{\text {Model }}\left\{\left(2 h_{e}^{\text {Model }}-1\right) p^{\prime}\left(1-p^{\prime}\right)+p^{\prime}\right\}\right] d p^{\prime}}{\int_{0}^{1} \exp \left[-2 N_{e}^{\text {Model }} s_{e}^{\text {Model }}\left\{\left(2 h_{e}^{\text {Model }}-1\right) p^{\prime}\left(1-p^{\prime}\right)+p^{\prime}\right\}\right] d p^{\prime}} .
$$

214 Assuming an initially rare and additive mutation $(h=1 / 2)$ with weak positive selection in a

215 large population $\left(s_{e}^{\text {Model }} N_{e}^{\text {Model }} p_{0}^{\text {Model }} \approx 0\right.$ and $\left.s_{e}^{\text {Model }} N_{e}^{\text {Model }} \gg 1\right)$, we obtain the classic 216 approximation, $u\left(p_{0}^{\text {Model }}\right) \approx 2 s_{e}^{\text {Model }} N_{e}^{\text {Model }} p_{0}^{\text {Model }}$, which upon substituting from Eq. 7 yields:

$$
u\left(p_{0}^{\text {Model }}\right) \approx \frac{2 \hat{\rho}_{H}^{\text {Model }} \hat{\rho}_{D}^{\text {Model }}}{\left(\hat{\rho}_{H}^{\text {Model }}+2 \hat{\rho}_{D}^{\text {Model }}\right)\left[\left(c_{D}^{\text {Model }}\right)^{2} \hat{\rho}_{H}^{\text {Model }}+2\left(c_{H}^{\text {Model }}\right)^{2} \hat{\rho}_{D}^{\text {Model }}\right]} 2 s_{\text {ave }}^{\text {Model }} .
$$

217 For example, because haploids and diploids have the same reproductive values in the obligately 218 sexual case $\left(\hat{c}_{H}^{\text {Model }}=\hat{c}_{D}^{\text {Model }}=1 / 2\right)$, we obtain: 


$$
u\left(p_{0}^{\text {Model }}\right) \approx \frac{8 \hat{\rho}_{H}^{\text {Model }} \hat{\rho}_{D}^{\text {Model }}}{\left(\hat{\rho}_{H}^{\text {Model }}+2 \hat{\rho}_{D}^{\text {Model }}\right)^{2}} 2 s_{\text {ave }}^{\text {Model }}
$$

219 (Eq. 13a in Bessho and Otto 2017), or simply $u\left(p_{0}^{\text {Model }}\right) \approx 2 s_{\text {ave }}^{\text {Model }}$ if haploid and diploid 220 population sizes are equal in terms of number of chromosomes $\left(\hat{\rho}_{H}^{\text {Model }}=2 / 3\right)$.

221 In the next three sections, we explore the implications of these results for the evolution 222 of haploid-diploid populations.

224 3.4. Effective selection in a haploid-diploid population

225 The strength of selection averaged across haploids and diploids, $S_{\text {ave }}^{\text {Model }}$, plays a key

226 role in the evolution of haploid-diploid populations. When a mutation is rare, both the rate of

227 change in allele frequency (Eq. 3b) and the approximate fixation probability (Eq. 10a) are

228 proportional to $s_{\text {ave }}^{\text {Model }}$. We thus begin by exploring how $s_{\text {ave }}^{\text {Model }}$ varies as we alter the amount

229 of asexual reproduction in haploid and diploid phases. We focus on the case where the mutation

230 does not affect fertilization success $\left(s_{M}^{f}=0\right)$, so that the average selection becomes:

$$
S_{\text {ave }}^{\text {Model }}=c_{H}^{\text {Model }} S_{M}^{w}+c_{D}^{\text {Model }} S_{R M}^{w}
$$

231 in both global and local regulation models (Eqs. (4) and (6)).

The relative evolutionary importance of selection in the haploid and diploid phases is

233 thus determined by the class reproductive values, $c_{H}^{\text {Model }}$ and $c_{D}^{\text {Model }}$ (where $c_{H}^{\text {Model }}+$

$234 c_{D}^{\text {Model }}=1$ ). Figures 2 (global regulation) and 3 (local regulation) illustrate the proportional

235 reproductive value of haploids, $c_{H}^{\text {Model }}$, as a function of the degree of asexual reproduction in

236 haploids (x-axis) and diploids (ranging from 0.05 in red to 0.95 in blue). With global regulation,

237 the frequency of haploidy within the population, $\hat{\rho}_{H}^{G}$ (given by Eq. A.4), varies with the

238 parameters (see inset graphs in Figure 2), rising with the frequency of haploid asexuality (x-axis

239 in inset) but declining with more asexuality in diploids (from red to blue). By contrast, with local 
240 regulation, the frequency of haploidy is held fixed by the strict density dependent competition

241 that we have assumed $\left(\hat{\rho}_{H}^{L}=0.8\right.$ in Figure $3(\mathrm{a})(\mathrm{b})$ and 0.3 in $\left.3(\mathrm{c})(\mathrm{d})\right)$.

242 In the left panels, haploids have a higher fertility $\left(w_{R} / w_{R R}=5\right)$, leading to a higher

243 haploid reproductive value, $c_{H}^{\text {Model }}$, especially with local regulation when haploids are also more

244 common $\left(\hat{\rho}_{H}^{L}=0.8\right.$ in Figure $\left.3 a\right)$. In the right panels, diploids have a higher fertility $\left(w_{R R} / w_{R}=\right.$

245 5), leading to a lower haploid reproductive value, especially when haploids are rare $\left(\hat{\rho}_{H}^{L}=0.3\right.$ in

246 Figure 3d).

When haploids are primarily sexual $\left(a_{H} \approx 0\right)$, increasing asexuality of the haploid

248 stage typically causes the reproductive value of haploids to rise, unless diploids are fitter and

249 more frequent (Figure $3 \mathrm{~d}$ and blue curves in Figure $2 \mathrm{~b}$ ). At the other extreme, the reproductive

250 value of haploids typically plummets to zero as haploid reproduction becomes primarily asexual

$251\left(a_{H} \approx 1\right)$ while diploids remain sexual, particularly with local regulation (Figure 3 ), because

252 haploids then act as a genetic "sink" contributing little to the diploid sub-population. This

253 downward trend when haploids are predominantly asexual is also seen with global regulation if

254 diploids are more fit (Figure 2b), except when the diploid population does not sustain itself and

255 goes extinct, which occurs when $a_{D}<0.2$ and $a_{H}=1$. The net result can thus be

256 non-monotonic (purple curves with $0.2<a_{D}<0.4$ in Figure $2 \mathrm{~b}$ and Figure 3(a)(b)(c)).

258 3.4. Effective population size in a haploid-diploid population

260 of asexuality. Figure 4 plots the effective population size (Eq. 8) relative to the total population

261 size, $N_{e}^{\text {Model }} / N_{t o t}$, as a function of the frequency of haploids, $\hat{\rho}_{H}^{\text {Model }}$ (x-axis), and the class

262 reproductive values (with $c_{H}^{\text {Model }}$ ranging from 0.05 in blue to 0.95 in red). As noted by Bessho 263 and Otto (2017), the effective population size is highest - and drift weakest - at intermediate 
264 frequencies of haploids and diploids, which ensures the least sampling error as organisms 265 alternate generations.

266 When haploids and diploids have equal reproductive values, as in the fully sexual case

$267\left(c_{H}^{\text {Model }}=c_{D}^{\text {Model }}=1 / 2\right)$, the effective population size is maximized at $\hat{\rho}_{H}^{\text {Model }} \approx 0.586$. With

268 asexual reproduction, the peak shifts towards whichever ploidy level has the higher reproductive

269 value. For example, if haploids have a high reproductive value (red) then the effective population

270 size is maximized at a higher frequency of haploids, reducing the amount of genetic drift in that

271 phase. Although not illustrated, the peak shifts to $N_{e}^{\text {Model }}=\hat{\rho}_{D}^{\text {Model }} N_{\text {tot }}$ when future

272 populations descend only from diploids $\left(c_{H}^{\text {Model }}=0\right)$ and to $N_{e}^{\text {Model }}=\left(\hat{\rho}_{H}^{\text {Model }} / 2\right) N_{\text {tot }}$ when

273 future populations descend only from haploids $\left(c_{H}^{\text {Model }}=1\right)$, effectively becoming diplontic or

274 haplontic, respectively (with the $1 / 2$ arising because haploids have half the number of 275 chromosomes).

276 Of course, the reproductive values, as well as the frequency of haploids with global

277 population regulation $\left(\hat{\rho}_{H}^{G}\right)$, depend in turn on the fitness parameters and the extent of asexuality,

278 as explored in the previous section. Figures 5 (global regulation) and 6 (local regulation)

279 illustrate the effective population size as a function of the frequency of haploid asexuality, $a_{H}$

280 (x-axis), and the frequency of diploid asexuality ( $a_{D}$ rising from red to blue), using the

281 parameters in Figures 2 and 3, respectively. The trends are often non-monotonic, with $N_{e}^{M o d e l} /$

$282 N_{\text {tot }}$ values varying around $1 / 2$ when the parameter values are intermediate. The effective

283 population size is often higher when diploids rarely reproduce asexually (red) rather than when

284 they frequently do (blue), although there are exceptions (particularly when the fitness and

285 frequency of diploids is high).

287 3.6. Fixation probability in a haploid-diploid population 
289 probability of a newly arisen mutation in a haploid-diploid population. When simulating the

290 global regulation model, we assumed that the population has reached the demographic

291 equilibrium, $\hat{\rho}_{H}^{G} N_{t o t}$ haploids and $\hat{\rho}_{D}^{G} N_{t o t}$ diploids (see Appendix A). We then chose one

292 resident allele $R$ at random and replaced it with a mutant allele $M$. After mutation, offspring were

293 sampled from the parental generation according to a multinomial distribution with expected

294 frequencies given by $x_{(G T)}$, repeating until the mutant allele fixed or was lost from the

295 population. We estimated the fixation probability as the fraction of 10,000 replicate simulations

296 leading to fixation.

297 We here consider the additive case $\left(h=1 / 2\right.$ and $\left.h_{e}=1 / 2\right)$, where the fixation

298 probability (Eq. 8) simplifies to:

$$
u\left(p_{0}^{\text {Model }}\right)=\frac{\exp \left[-2 p_{0}^{M o d e l} N_{e}^{M o d e l} S_{e}^{M o d e l}\right]-1}{\exp \left[-2 N_{e}^{M o d e l} s_{e}^{M o d e l}\right]-1}
$$

299 and where $s_{e}^{\text {Model }}=2 s_{\text {ave }}^{\text {Model }}$ (Eq. 6). Figure 7 plots the fixation probability as a function of the 300 average selection pressure, $s_{\text {ave }}^{\text {Model }}$, when the reproductive values and chromosome numbers in 301 haploids and diploids are equal $\left(c_{H}^{\text {Model }}=c_{D}^{\text {Model }}\right.$ and $\left.\hat{\rho}_{H}^{\text {Model }}=2 / 3\right)$ and $s_{\text {ave }}^{\text {Model }}=[(1 /$

$3022 s M w+1 / 2 s R M w$. The diffusion Eq. (11) provides an excellent fit, as does the approximation

303 Eq. (9b) for selection coefficients that are positive and not too weak. In this case, the results are 304 the same with global and local population regulation (Fig. 7a and 7b, respectively) and are 305 insensitive to how much selection occurs in the haploid or diploid phases $\left(s_{M}^{M o d e l}\right.$ and $s_{R M}^{M o d e l}$, 306 respectively), as long as $s_{\text {ave }}^{\text {Model }}$ is held constant (see additional simulations in Supplementary 307 Mathematica file). As expected, the extent of selection in the haploid versus diploid phase 308 matters more when the mutation is not additive $\left(h \neq 1 / 2\right.$ and $\left.h_{e} \neq 1 / 2\right)$ (supplementary 309 Mathematica file). 
311 degree of asexuality $\left(a_{H}\right.$ and $\left.a_{D}\right)$ when the population size is globally (Fig. 8) or locally (Fig. 9)

312 regulated, assuming only selection in haploids or only in diploids. For example, with additive

313 mutations, the fixation probability can be approximated as $u \approx 4 c_{H}^{M o d e l} N_{e}^{M o d e l} p_{0}^{M o d e l} s_{M}^{w}$ when

314 selection occurs only in the haploid phase or $u \approx 4 c_{D}^{\text {Model }} N_{e}^{\text {Model }} p_{0}^{\text {Model }} s_{R M}^{w}$ with selection only

315 in the diploid phase, indicating that the fate of mutations depends as much on the strength of

316 selection as on the reproductive value of the ploidy phase in which selection acts (as illustrated in

317 Fig. 2 and 3). Figures 8 (global) and 9 (local) illustrate how the fixation probability depends on

318 the various parameters in the model, particularly the amount of asexual reproduction in haploids

319 (x-axis) and diploids ( $a_{D}$ rising from red to blue). The trends can be understood by the combined

320 effects of the parameters on the reproductive value and the effective population size (e.g., Fig.

3219 (a) is proportional to the product of Fig. 3(a) and Fig. 6(c)).

\section{4. Discussion}

Across the phylogenetic tree of life, organisms have diverse and complex reproductive

325 strategies (Bell 1982). Classical population genetic theory has, however, focused most on fully

326 haploid or diploid life cycles with obligate sexuality. In this article we develop a stochastic

327 model for the population genetics of haploid-diploid organisms considering demography,

328 asexuality, and habitat differentiation between haploid and diploid stages. Using a separation of

329 time scales, we derive a diffusion approximation for the change in allele frequency, allowing us

330 to estimate the fixation probability of new mutations, the effective strengths of selection and

331 dominance, as well as the effective population size of haploid-diploid populations.

333 5.1. Natural selection in a haploid-diploid population

334 Our results indicate that the strength of natural selection and the extent of genetic drift 
335 depend strongly on the reproductive value of haploid versus diploid phases. In the simplest case,

336 when the effect of a mutation is weak, additive, positive, and absent in the gamete stage $\left(s_{M}^{f}=0\right)$,

337 the fixation probability is proportional to the effective strength of selection (Eq. 10), $s_{e}^{\text {Model }}=$

$3382 s_{\text {ave }}^{\text {Model }}$, which in turn is proportional to the amount of selection in and the reproductive value of

339 haploids and diploids (Eqs. 2, and 5).

These analytical results reveal some evolutionary principles for populations that

341 undergo an alternation of generations. One consequence is that the balance of opposing selection

342 pressures in haploids and diploids (Eqs. 4 and 6) depends not only on the selection coefficients,

343 but also on the relative reproductive values of haploids $\left(c_{H}^{M o d e l}\right)$ versus diploids $\left(c_{D}^{\text {Model }}\right)$. Thus,

344 the very direction of evolution depends on the extent of asexuality in the two phases and the

345 relative survival and fertility of haploids versus diploids when there is "ploidally antagonistic

346 selection" (Immler et al. 2012).

347 The efficacy of selection to fine tune traits in haploids and diploids also depends on the

348 class reproductive values. For example, when the population is regulated by local density

349 dependence (i.e., the haploid and diploid phases are spatially or temporally distinct), higher

350 reproductive success in haploids increases the efficiency of haploid selection (compare Figure 3a

351 to $3 \mathrm{~b})$. However, when there is extremely rare sexuality in haploids $\left(a_{H}\right.$ near one), diploid

352 selection tends to be more effective because of increasing competition between offspring from

353 haploids. By contrast, the trends differ with global density dependence (e.g., species that are

354 more isomorphic with small ecological differences between stages). For example, the

355 reproductive value of haploids remains high even when they reproduce primarily asexually in the

356 global regulation model (see Figure 2 when $a_{H}$ approaches one), because haploids then make up

357 a larger proportion of the total population size (see inset figures). Thus, whether selection is

358 effective in the haploid phase when that phase mainly reproduces asexually is quite sensitive to 
359 the nature of competition.

360 Our work can also be useful in the design of field studies and the interpretation of data

361 for species that alternate generations. To understand the efficiency of selection on haploid and

362 diploid phases, we not only need data about the fraction of haploids and diploids and their

363 fertility and mortality (e.g., Thornber and Gains 2004; Vieira et al., 2018a; Vieira et al. 2018b),

364 but we also need to know about the extent of asexuality in each phase and whether they compete

365 for common or different resources.

367 5.2. Genetic drift and effective size

368 The impact of random genetic drift on the genetic diversity of haploid-diploid 369 population depends on the effective population size (Eq. 7). As we had found previously in a 370 haploid-diploid model with obligate sexuality (Bessho and Otto 2017, pp. 431), the effective 371 population size with asexuality is generally smaller than the total number of individuals and 372 again depends strongly on the reproductive value of each phase (Figures 4-6). With obligate 373 sexuality, the reproductive values of haploids and diploids are equal, and the effective population 374 size is maximized (drift minimized) when haploids comprise $2 / 3$ of the population, making the 375 number of chromosomes equal between haploids and diploids. Asexual reproduction, however, 376 causes the reproductive value of haploids and diploids to differ (Eqs. 2 and 5). Consequently, 377 drift is lessened if the phase with the higher reproductive value is more common (see shifts in 378 peaks in Figure 4).

\section{$380 \quad$ 5.3. "Ploidally-structured" population}

382 patch dynamics play in two-patch models of evolution. In a spatially structured population, 
383 subdivided local populations are genetically connected by migration. A haploid-diploid system

384 can be seen as being ploidally structured, where gene flow describes the movement of alleles

385 through sexual reproduction, with meiosis causing flow to haploidy and syngamy flow to

386 diploidy. We note that our research reveals that all qualitative results are equally accurate for

387 evolution in a two-patch system (see Supplementary Mathematica File). For example, fixation

388 probability strongly depends on class reproductive values of each patch.

This analogy suggests an interesting idea: complex reproductive systems can be

390 considered and analyzed using the tools of metapopulation theory. For example, many

391 eukaryotes including terrestrial plants, insects, and fishes, often exhibit ploidy variation,

392 including polyploid members (Otto and Whitton 2000; Comai 2005). In such species, individuals

393 characterized by different numbers of chromosomes coexist, with complex reproductive

394 relationships causing gene flow between them (Ramsey and Schemske 1998). Similarly, social

395 insects often exhibit complex sex determination systems linked with ploidy levels

396 (haplodiploidy).

397 Our research suggests that these ploidally-structured populations can be fruitfully

398 treated as metapopulations. Selection and drift in populations with diploids, triploids, and

399 tetraploids can, for example, be considered as a three-patch model. In this system, we conjecture

400 that the average strength of selection that is evolutionarily relevant would be the mean selection

401 coefficient in each ploidy class, weighted by its class reproductive value, with additional terms

402 coming from reproductive interactions (akin to the term of $s_{M}^{f}$ in Eqs. 4).

403 Many evolutionary aspects of haploid-diploid populations remain to be investigated.

404 One avenue that we are exploring is how model parameters can be estimated from field data. For

405 example, the analogy between spatially and ploidally structured population suggests that genetic

406 differences between haploids and diploid can be used to estimate gene flow between them (i.e., 
407 rates of sex), akin to using Fst to inform estimates of migration (e.g., Slatkin 1987). Another

408 fruitful avenue for further work is to determine how fluctuations in population size affect the

409 effective population size of species that alternate generations. In classical population genetics

410 theory, such fluctuations can be captured by using the harmonic mean population in place of the

411 total population size (Karlin 1968). It is unclear, however, whether the same is true in

412 haploid-diploid populations. Can the harmonic total population size simply replace $N_{t o t}$ in the

413 global model of population regulation? Similarly, can the harmonic population sizes of haploids

414 and diploids replace $N_{H}$ and $N_{D}$ with local regulation? The answer is unclear because

415 population size fluctuations perturb the fast ecological dynamics away from the steady state

416 (especially $\hat{\rho}_{H}^{\text {Model }}$ ), and the impact of these perturbations on selection and drift is unknown.

417 Further research is needed to clarify evolutionary processes in the wide variety of species that

418 alternate generations.

420 Acknowledgements

421 We here thank Alireza Ghaseminejad for help in developing our manuscript. We also thank $\mathrm{H}$.

422 Ohtsuki and Y. Uchiumi for the helpful comment.

\section{Funding sources}

425 This project was funded by a Grand-in-Aid from a Japan Society for the Promotion of Science 426 (JSPS) to KB (16J05204) and by a Discovery grant from the Natural Sciences and Engineering

427 Research Council of Canada (NSERC RGPIN-2016-03711) to S.P.O. 
431 A.1. Equilibrium with global regulation

432 We derive the fixation probability in a haploid-diploid population using a diffusion

433 approximation (e.g., Bessho and Otto 2017). We first derive the stable equilibrium in the global

434 regulation model, allowing for asexual reproduction in each phase. In the Wright-Fisher model,

435 all individuals reproduce and then the parents die (non-overlapping generations). Let $b_{(G T)}$

436 represent the number of reproductive cells of each type in the next generation:

$$
\begin{aligned}
& b_{R}=\left(1-a_{D}\right) w_{R R} x_{R R}+\left(1-a_{D}\right) \frac{w_{R M} x_{R M}}{2}+a_{H} w_{R} x_{R}, \\
& b_{M}=\left(1-a_{D}\right) \frac{w_{R M} x_{R M}}{2}+\left(1-a_{D}\right) w_{M M} x_{M M}+a_{H} w_{M} x_{M}, \\
& b_{R R}=\left(1-a_{H}\right) \frac{f_{R}}{2} \frac{w_{R}^{2} x_{R}^{2}}{w_{R} x_{R}+w_{M} x_{M}}+a_{D} w_{R R} x_{R R}, \\
& b_{R M}=\left(1-a_{H}\right) \frac{f_{R}+f_{M}}{2} \frac{w_{R} w_{M} x_{R} x_{M}}{w_{R} x_{R}+w_{M} x_{M}}+a_{D} w_{R M} x_{R M}, \\
& b_{M M}=\left(1-a_{H}\right) \frac{f_{M}}{2} \frac{w_{M}^{2} x_{M}^{2}}{w_{R} x_{R}+w_{M} x_{M}}+a_{D} w_{M M} x_{M M} .
\end{aligned}
$$

437 The probability that a reproductive cell of genotype $(G T)$ is sampled from the offspring produced

438 by the previous generation of adults is

$$
q_{(G T)}=\frac{b_{(G T)}}{b_{R}+b_{M}+b_{R R}+b_{R M}+b_{M M}} .
$$

439 Therefore, the composition of offspring in the next generation is given by the multinomial

440 distribution, sampling $N_{t o t}$ individuals in proportion to Eq. (A2). Using Eq. (A1) and (A2), we

441 describe the conditional expectation of change in the number of individuals of genotype (GT),

$442 \Delta X_{(G T)}(t)=X_{(G T)}(t+1)-X_{(G T)}(t)$, as

$$
\mathrm{E}\left[\Delta X_{(G T)}(t) \mid \vec{X}(t)=\vec{x}\right]=N_{t o t} q_{(G T)}-x_{(G T)},
$$


443 where $\mathrm{E}\left[\Delta F\left(X_{(G T)}(t)\right) \mid \vec{X}(t)=\vec{x}\right]$ is the conditional expected value for change in the function

$444 \quad F$ of the random variable given that $\vec{X}(t)=\left(\begin{array}{lllll}X_{R}(t) & \left.X_{M}(t) \quad X_{R R}(t) \quad X_{R M}(t) \quad X_{M M}(t)\right)^{T}\end{array}\right.$

445 equals $\vec{x}=\left(\begin{array}{lllll}x_{R} & x_{M} & x_{R R} & x_{R M} & x_{M M}\end{array}\right)^{T}$.

446 To simplify this fully stochastic system, we assume that the resident population is large

447 and treat demographic changes deterministically prior to the appearance of the mutation.

448 Considering the dynamics of the resident population, we then find the equilibrium of these

449 dynamical equations by solving $N_{t o t} q_{R}-\hat{x}_{R}=0$ and $N_{t o t} q_{R R}-\hat{x}_{R R}=0\left(\hat{x}_{R}+\hat{x}_{R R}=N_{t o t}\right)$.

450 Setting $\hat{x}_{R}=\hat{\rho}_{H}^{G} N_{\text {tot }}$ and $\hat{x}_{R R}=\hat{\rho}_{D}^{G} N_{\text {tot }}$, the fraction of haploids $\hat{\rho}_{H}^{G}$ (and diploids $\hat{\rho}_{D}^{G}=1-$

$451 \hat{\rho}_{H}^{G}$ ) at equilibrium becomes,

$$
\hat{\rho}_{H}^{G}=\frac{a_{H} w_{R}+a_{D} w_{R R}-2 w_{R R}+\sqrt{4\left(1-a_{H}\right)\left(1-a_{D}\right) \widetilde{w}_{R} w_{R R}+\left(a_{H} w_{R}-a_{D} w_{R R}\right)^{2}}}{2\left[a_{H} w_{R}+\left(1-a_{H}\right) \widetilde{w}_{R}-w_{R R}\right]},
$$

452 where $\widetilde{w}_{R}=f_{R} w_{R} / 2$ is the fitness of haploids considering the cost of sex (see Supplementary

453 Mathematica file for the step-by-step derivation). We note that, when the fertility of haploids is

454 much greater than that of diploids $\left(w_{R} \gg w_{R R}\right)$, the frequency of haploids in a population

455 approaches $a_{H} /\left\{a_{H}+\left[\left(1-a_{H}\right) f_{R} / 2\right]\right\}$, which is less than one because sexual reproduction of

456 the haploids produces diploids (the $\left(1-a_{H}\right) f_{R} / 2$ term). Conversely, when the fertility of

457 diploids is much greater than haploids $\left(w_{R} \ll w_{R R}\right)$, the frequency of haploids approaches

$4581-a_{D}$, the rate at which diploids undergo meiosis.

460 A.2. First moment of change in allele frequency

461 To derive the first moment of change in allele frequency, $m^{\text {Model }}\left(p_{\text {ave }}\right)$, we apply a

462 separation of time scales (e.g., Nagylaki 1976; Otto and Day 2007; Bessho and Otto 2017).

463 Details of the calculation are represented in the Supplementary Mathematica file. We first

464 transform the expected change in the number of individuals of each type (five variables that sum 
465 to $N_{t o t}$ ) into the expected change in a new set of four variables, $\Theta \in\left\{p_{\text {ave }}, \delta_{p}, \eta_{H W}, \rho_{H}\right\}$,

466 described by the functions:

$$
\mathrm{E}[\Delta \Theta \mid \vec{X}(t)=\vec{x}]=f_{\Theta}^{\text {Model }}\left(\epsilon, p_{\text {ave }}, \vec{\theta}\right)
$$

467 where $\vec{\theta}=\left(\delta_{p}, \eta_{H W}, \rho_{H}\right)$ and $\epsilon$ is proportional to the selection coefficients and assumed small

468 (the functions $f$ are given explicitly in the Supplementary Mathematica file). With local

469 regulation, $\rho_{H}$ is assumed fixed at $N_{H} / N_{\text {tot }}$ and dropped from the variable set, $\Theta$.

470 To constant order (setting the small changes due to selection to zero, $\epsilon \rightarrow 0$ ), the fast

471 ecological dynamics of the system are described by: $f_{\Theta}^{\text {Model }}\left(0, p_{\text {ave }}, \vec{\theta}\right)$. This system of

472 equations rapidly approaches a steady state found by solving $f_{\Theta}^{\text {Model }}\left(0, p_{\text {ave }}, \vec{\theta}\right)=0$, which

473 gives $\bar{\delta}_{p}=\bar{\eta}_{H W}=0$, and $\bar{\rho}_{H}=\hat{\rho}_{H}^{G}$ (Eq. A.4). To this order, the steady state change in allele

474 frequency is zero, $f_{p_{\text {ave }}}^{\text {Model }}\left(0, p_{\text {ave }}, \vec{\theta}\right)=0$. We then describe slower changes, including changes

475 in allele frequency due to selection, by describing the deviations that occur around this steady

476 state. Specifically, to order $\epsilon$, the variables are allowed to deviate from the steady state by

$477 \delta_{p}=\tilde{\delta}_{p} \epsilon, \eta_{H W}=\tilde{\eta}_{H W} \epsilon$, and $\rho_{H}=\hat{\rho}_{H}^{G}+\tilde{\rho}_{H} \epsilon$, and the dynamics $f_{\Theta}^{\text {Model }}\left(\epsilon, p_{\text {ave }}, \vec{\theta}\right)$ are then

478 approximated using a Taylor series expansion. Defining the average allele frequency by

479 combining haploid and diploid populations using an arbitrary weighting, $p_{a v e}=\omega p_{H}+$

$480(1-\omega) p_{D}$, we show in the Supplementary Mathematica file that setting the weights proportional

481 to the class reproductive values (given by Eq. (2) with global regulation and Eq. (5) with local

482 regulation) is the only choice that separates evolutionary change in $p_{\text {ave }}$ from changes in the

483 other variables to order $\epsilon$. Defining the average allele frequency in this way (Eq. 1a) and taking

484 the Taylor series, the change in allele frequency becomes:

$$
\begin{aligned}
\mathrm{E}\left[\Delta p_{\text {ave }} \mid \vec{X}(t)=\vec{x}\right] & \approx M^{\text {Model }}\left(p_{\text {ave }}\right) \\
& =\frac{p_{\text {ave }}\left(1-p_{\text {ave }}\right)}{2}\left[2 s_{\text {ave }}^{\text {Model }}+2 c_{D}^{\text {Model }} p_{\text {ave }}(1-2 h) s_{M M}^{W}\right]
\end{aligned}
$$


486 A.3. Second moment of change in average allele frequency

488 haploid-diploid population with asexuality. We again assume that the population size is very

489 large, that selection is very weak, and that the system has approached the steady state in

$490\left(\delta_{p}, \eta_{H W}, \rho_{H}\right)$, ignoring deviations that are of $O(\epsilon)$. Because selection is assumed weak, the

491 second moment is well approximated by that of the neutral model (to constant order, $\epsilon \rightarrow 0$ ).

493 both the global and local regulation models, and we can sample the haploid offspring according

494 to a binomial distribution, with expectation and variance: $\mathrm{E}\left[X_{M} \mid \vec{X}(t)=\vec{x}\right]=q_{M} N_{H}$ and

$495 \operatorname{Var}\left[X_{M} \mid \vec{X}(t)=\vec{x}\right]=q_{M}\left(1-q_{M}\right) N_{H}$ where $q_{M}=b_{M} /\left(b_{R}+b_{M}\right)$. To simplify the equation, we

496 set $\mathrm{E}\left[X_{(G T)} \mid \vec{X}(t)=\vec{x}\right]=m_{(G T)}$ and $\operatorname{Var}\left[X_{(G T)} \mid \vec{X}(t)=\vec{x}\right]=v_{(G T)}$, finding that:

$$
\begin{aligned}
& \mathrm{E}\left[\Delta X_{M} \mid \vec{X}(t)=\vec{x}\right]=m_{M}-x_{M}, \\
& \mathrm{E}\left[\left(\Delta X_{M}\right)^{2} \mid \vec{X}(t)=\vec{x}\right]=v_{M}+m_{M}^{2}-2 m_{M} x_{M}+x_{M}^{2} .
\end{aligned}
$$

497 In terms of allele frequencies (rather than numbers), we have the first and second moments for

498 the haploid offspring population, $\mathrm{E}\left[\frac{\Delta X_{M}}{N_{H}} \mid \vec{X}(t)=\vec{x}\right]=\frac{1}{N_{H}} \mathrm{E}\left[\Delta X_{M} \mid \vec{X}(t)=\vec{x}\right]$ and

$$
\mathrm{E}\left[\left(\frac{\Delta X_{M}}{N_{H}}\right)^{2} \mid \vec{X}(t)=\vec{x}\right]=\frac{1}{N_{H}^{2}} \mathrm{E}\left[\left(\Delta X_{M}\right)^{2} \mid \vec{X}(t)=\vec{x}\right]
$$

501 expectation, variance, and covariance: $m_{(G T)}=q_{(G T)} N_{D}, v_{(G T)}=q_{(G T)}\left(1-q_{(G T)}\right) N_{D}$, and

$502 \operatorname{Cov}\left[X_{R M}, X_{M M} \mid \vec{X}(t)=\vec{x}\right]=q_{R M} q_{M M} N_{D}$, where $q_{(G T)}=b_{(G T)} /\left(b_{R R}+b_{R M}+b_{M M}\right)$. To derive

503 the moments of the allele frequency in diploids, we define, $y_{M}=\left(x_{R M} / 2\right)+x_{M M}$ and

$504 Y_{M}=\left(X_{R M} / 2\right)+X_{M M}$. The moments of random variable $Y$ are then: 


$$
\begin{aligned}
& \mathrm{E}\left[Y_{M} \mid \vec{X}(t)=\vec{x}\right]=\frac{m_{R M}}{2}+m_{M M}, \\
& \mathrm{E}\left[Y_{M}^{2} \mid \vec{X}(t)=\vec{x}\right]=\frac{v_{R M}+m_{R M}^{2}}{4}+\left(\operatorname{Cov}\left[X_{R M}, X_{M M} \mid \vec{X}(t)=\vec{x}\right]+m_{R M} m_{M M}\right)+\left(v_{M M}+m_{M M}^{2}\right), \\
& \mathrm{E}\left[\Delta Y_{M} \mid \vec{X}(t)=\vec{x}\right]=\frac{\mathrm{E}\left[\Delta X_{R M} \mid \vec{X}(t)=\vec{x}\right]}{2}+\mathrm{E}\left[\Delta X_{M M} \mid \vec{X}(t)=\vec{x}\right]-y_{M}, \\
& \mathrm{E}\left[\left(\Delta Y_{M}\right)^{2} \mid \vec{X}(t)=\vec{x}\right]=\mathrm{E}\left[Y_{M}^{2} \mid \vec{X}(t)=\vec{x}\right]-2 \mathrm{E}\left[Y_{M} \mid \vec{X}(t)=\vec{x}\right] y_{M}+y_{M}^{2} .
\end{aligned}
$$

506 define $Z_{M}=c_{H}^{\text {Model }} \frac{X_{M}}{N_{H}}+c_{D}^{M o d e l} \frac{Y_{M}}{N_{D}}$ and consider the expectation of change in this random

507 variable. Plugging in Eqs. (7a), (7b), (8c), and (8d), we have

$$
\mathrm{E}\left[\left(\Delta Z_{M}\right)^{2} \mid \vec{X}(t)=\vec{x}\right]=\frac{p_{\text {ave }}\left(1-p_{\text {ave }}\right)\left[\left(c_{D}^{\text {Model }}\right)^{2} \hat{\rho}_{H}^{\text {Model }}+\left(c_{H}^{\text {Model }}\right)^{2}\left(2 \hat{\rho}_{D}^{\text {Model }}\right)\right]}{\hat{\rho}_{H}^{\text {Model }}\left(2 \hat{\rho}_{D}^{\text {Model }}\right) N_{\text {tot }}} .
$$

508 After transforming time scales using the variable $\tau=t / N_{t o t}$ and defining $P(\tau)=Z\left(N_{t o t} \tau\right)$,

509 we have the diffusion coefficient $v^{\text {Model }}\left(p_{\text {ave }}\right)=\lim _{N_{t o t} \rightarrow \infty} \mathrm{E}\left[\frac{(P(\tau+\Delta \tau)-P(\tau))^{2}}{\Delta \tau}\right]$ by taking the

510 limit $N_{\text {tot }} \rightarrow \infty$, giving Eq. (3c). Similarly, we derive the drift coefficient using Eq. (A6)

$511 \quad\left(m^{\text {Model }}=M^{\text {Model }} N_{\text {tot }}\right)$, giving Eq. (3b).

\section{References}

514 Bell, G. 1982. The Masterpiece of Nature: The Genetics and Evolution of Sexuality. University 515 of California Press. Berkeley, CA.

516 Bell, G., 1994. The comparative biology of the alternation of generations. Lectures on 517 mathematics in the life sciences $25,1-26$.

518 Bessho, K., Otto, S. P. 2017. Fixation probability in a haploid-diploid population. Genetics 205, 519 421-440.

520 Comai, L. 2005. The advantages and disadvantages of being polyploid. Nature Reviews Genetics $5216,836-846$.

522 Coelho, S. M., Peters, A. F., Charrier, B., Roze, D., Destombe, C., Valero, M., Cock, J. M., 2007.

523 Complex life cycles of multicellular eukaryotes: new approaches based on the use of 524 model organisms. Gene 406, 152-170. 
525 Crow, J. F., Kimura, M. (1970). An introduction to population genetics theory. The Blackburn

$526 \quad$ Press, New Jersey.

527 Deithier, M. N. (1981). Heteromorphic algal life histories: The seasonal pattern and response to

528 herbivory of the brown crust, Ralfsia californica. Oecologia 49, 333-339.

529 Destombe, C., Godin, J., Nocher, M., Richerd, S., Valero, M. (1993). Differences in response

530 between haploid and diploid isomorphic phases of Gracilaria verrucosa (Rhodophyta:

531 Gigartinales) exposed to artificial environmental conditions. Hydrobiologia 260/261,

532 131-137.

533 De Wreede, R. E., Klinger, T. 1988. Reproductive strategies in algae. Plant reproductive ecology:

$534 \quad$ Patterns and strategies, 267-284.

535 Dyck, L. J., De Wreede, R. E. (2006). Seasonal and spatial patterns of population density in the

536 marine macroalga Mazzaella splendens (Gigartinales, Rhodophyta). Phycological

$537 \quad$ Research, 54(1), 21-31.

538 Goldstein, D. B. 1992. Heterozygote advantage and the evolution of dominant diploid phase.

539 Genetics 132, 1195-1198.

540 Haghes, J. S., Otto, S. P. 1999. Ecology and the evolution of biphasic life cycles. The American

$541 \quad$ Naturalist 154, 306-330.

542 Hannach, G., Santelicesm, B. 1985. Ecological differences between the isomorphic reproductive

543 phases of two species of Iridaea (Rhodophyta: Gigartinales). Marine Ecology Progress

544 Series 22, 291-303.

545 Hawkes, M. W. 1990. Reproductive strategies. Biology of the red algae, 455-476.

546 Immler, S., G. Arnqvist, and S. P. Otto. 2012. Ploidally antagonistic selection maintains stable

547 genetic polymorphism. 66:55-65.

548 Jenkins, C. D., Kirkpatrick, M., 1995. Deleterious mutation and the evolution of genetic life

$549 \quad$ cycles. Evolution, 512-520.

550 Karlin, S. 1968. Rates of approach to homozygosity for finite stochastic models with variable

551 population size. The American Natualist 102, 443-455.

552 Kimura, M., 1957. Some problems of stochastic processes in genetics. The Annals of

553 Mathematical Statistics, 882-901.

554 Kimura, M., 1962. On the probability of fixation of mutant genes in a population. Genetics 47 , 555713.

556 Mable, K. B., Otto, P. S., 1998. The evolution of life cycles with haploid and diploid phases.

557 BioEssays 20, 453-462.

558 Nagylaki, T., 1976. The evolution of one-and two-locus systems. Genetics 83, 583-600.

559 Orr, H. A., Otto, S. P. 1994. Does diploidy increase the rate of adaptation? Genetics 136, 560 1475-1480. 
561 Otto, S., Goldstein, D., 1992. Recombination and the evolution of diploidy. Genetics 131,

562

563

564

565

566

567

568

569

570

571

572

573

574

575

576

577

578

579

580

581

582

583

584

585

586

587

588

589

590

591

592

593

594

595

596 745-751.

Otto, S., 1994. The role of deleterious and beneficial mutations in the evolution of ploidy levels. Lectures on Mathematica in the Life Sciences 25, 69-98.

Otto, S. P., Marks, J. C. 1996. Mating system and the evolutionary transition between haploidy and diploidy. Biological Journal of the Linnean Society 57, 197-218.

Otto, S. P., Day, T., 2007. A biologist's guide to mathematical modeling in ecology and evolution. Princeton University Press.

Otto, S. P., Whitton, J. 2000. Polyploid incidence and evolution. Annu. Rev. Genet. 34, 401-437.

Perrot, V., Richerd, S., Valéro, M., 1991. Transition from haploidy to diploidy. Nature 351, 315-317.

Ramsey, J., Schemske, D. W. 1998. Pathways, mechanisms, and rates of polyploid formation in flowering plants. Annual reviews of Ecology and Systematics 29: 467-501.

Raper, J. R., Flexer, A. S. (1970). The road to diploidy with emphasis on a detour. In Symp. Soc. Gen. Microbiol (Vol. 20, pp. 401-432).

Rousset, F. (2004). Genetic structure and selection in subdivided populations. Princeton University Press, New Jersey.

Scott, M. F., Rescan, M. 2016. Evolution of haploid-diploid life cycles when haploid and diploid fitnesses are not equal. Evolution 71, 215-226.

Slatkin, M. 1987. Gene flow and the geographic struture of natural populations. Science New Series 236: 787-792.

Slocum C. J. 1980. Differential susceptibility to grazers in two phases of an intertidal alga: Advantages of heteromorphic generations. Journal of Experimental Marine Biology and Ecology 46, 99-110.

Taylor, P. D. 1980. Allele-Frequency Change in a Class-Structured Population. The American Naturalist 135, 95-106.

Thornber, C. S., Gains, S. D. 2004. Population demographics in species with biphasic life cycle. Ecology 85, 1661-1674.

Thornber, C., Stachowicz, J. J., Gains, S. 2006. Tissue type matters: selective herbivory on different life history stages of an isomorphic alga. Ecology 87, 2255-2263.

Vieira, V. M. N. C. S., Engelen, A. H., Huanel, O. R., Guillemin, M. 2018a. Haploid females in the isomorphic biphasic life-cycle of Gracilaria chilensis excel in survival. BMC Evolutionary Biology 18:174.

Vieira, V. M. N. C. S., Engelen, A. H., Huanel, O. R., Guillemin, M. 2018b. Differentiation of haploid and diploid fertilities in Gracilaria chilensis affect ploidy ratio. BMC Evolutionary Biology 18:183. 
597 Willson, M. F. 1981. On the evolution of complex life cycles in plants: a review and an ecological perspective. Annals of the Missouri Botanical Garden 68, 275-300.

\section{Figure Captions}

601 Fig. 1. An illustration of the haploid-diploid models. (a) In the global regulation model, both

602 haploids and diploids occupy the same habitat and density dependence holds the total population

603 size $N_{\text {tot }}$ constant. (b) In the local regulation model, each ploidy stage occupies a different habit,

604 therefore density dependence regulates the population size of haploids $\left(N_{H}\right)$ and diploids $\left(N_{D}\right)$

605 separately.

606

607 Fig. 2. Class reproductive value of haploids in the global regulation model, $c_{H}^{G}$. Curves show $c_{H}^{G}$

608 as a function of the degree of haploid asexuality, $a_{H}$ (x-axis), with the degree of diploid

609 asexuality ranging in color from $a_{D}=0.05$ (red) to 0.95 (blue) in increments of 0.05 . Other

610 parameters are set as: (a) $f_{R}=0.5, w_{R}=5000, w_{R R}=1000$, (b) $f_{R}=0.5, w_{R}=1000$,

$611 w_{R R}=5000$. The resulting frequency of haploids, $\hat{\rho}_{H}^{G}$ (Eq. A.4), is shown in the inset plots.

613 Fig. 3. Proportional reproductive value of haploids in the local regulation model, $c_{H}^{L}$. Parameters

614 are the same as Fig. 2, except that haploids are held fixed at a frequency of (a)(b) $\hat{\rho}_{H}^{L}=0.8$ or

615 (c)(d) $\hat{\rho}_{H}^{L}=0.3$. We consider the case when (a)(c) haploid fitness parameter is larger than

616 diploid $w_{R}=5000, w_{R R}=1000\left(w_{H}>w_{D}\right)$, and when (b)(d) diploid fitness parameter is

617 larger than haploid $w_{R}=1000, w_{R R}=5000\left(w_{H}<w_{D}\right)$.

619 Fig. 4. The effective population size of a haploid-diploid population. The relative effective

620 population size over the total population size $\left(N_{e}^{\text {Model }} / N_{t o t}\right.$, Eq. 7) is shown as a function of the

621 frequency of haploids $\left(\hat{\rho}_{H}^{\text {Model }}, \mathrm{x}\right.$-axis), when the haploid reproductive value $\left(c_{H}^{\text {Model }}\right)$ varies from 
6220.05 (blue) to 0.95 (red) in increments of 0.05 . This figure applies to both global and local

623 regulation models.

624

625 Fig. 5. The effective population size of a haploid-diploid population in the global regulation

626 model as a function of the degree of asexuality, $a_{H}$ and $a_{D}$. Parameters are the same as in Fig. 2

627 and determine the relative class reproductive values $\left(c_{H}^{G}\right)$ and fraction of haploids $\left(\hat{\rho}_{H}^{G}\right)$ according

628 to Eqs. (2a) and (A.4).

629

630 Fig. 6. The effective population size of a haploid-diploid population in the local regulation model.

631 Parameters are the same as in Fig. 3 and determine the relative class reproductive values $\left(c_{H}^{L}\right)$ by

632 Eqs. (5). The frequencies of haploids are held fixed at a frequency of (a)(b) $\hat{\rho}_{H}^{L}=0.9$, (c)(d)

$633 \hat{\rho}_{H}^{L}=0.8,(\mathrm{e})(\mathrm{f}) \hat{\rho}_{H}^{L}=0.6$, or $(\mathrm{g})(\mathrm{h}) \hat{\rho}_{H}^{L}=0.3$.

634

635 Fig. 7. Fixation probability given the average strength of selection, $s_{a v e}^{\text {Model }}$, for global (a) and

636 local (b) regulation models. The solid curve gives the analytical result from the diffusion

637 approximation (Eq. (11)) and the dashed curve gives the linear approximation (Eq. (9b)). Black

638 dots indicate the fixation probability estimated from 10000 numerical simulations with 95\% CI

639 (Wilson score interval for binomial). Parameters: $N_{t o t}=90, N_{H}=60, N_{D}=30, f_{R}=0.5$,

$640 w_{R}=w_{R R}=1000, a_{H}=a_{D}=0.1, h=0.5, s_{M}^{f}=0, s_{M}^{w}=s_{R M}^{w}=s_{a v e}^{\text {Model }}$, such that the

641 fraction of haploids in the resident population is $\hat{\rho}_{H}^{\text {Model }}=2 / 3$ and class reproductive values

642 are equal $c_{H}^{\text {Model }}=c_{D}^{\text {Model }}=1 / 2$. Holding $s_{\text {ave }}^{\text {Model }}=1 / 2\left(s_{M}^{w}+s_{R M}^{w}\right)$ constant, similar results

643 are obtained for a range of different choices of $s_{M}^{w}$ and $s_{R M}^{w}$ (see supplementary Mathematica

644 file).

645 
646 Fig. 8. The fixation probability in a haploid-diploid population in the global regulation model.

647 Curves gives the linear approximation for the fixation probability (Eq. (9a)). Parameters are the

648 same as in Fig. 2. Selection acts only in the haploid or diploid phase, with selection coefficients

649 set as $s_{M}^{w}=0.02$ for haploid selection (a)(b) and $s_{R M}^{w}=0.02$ for diploid selection (c)(d).

650

651 Fig. 9. The fixation probability in a haploid-diploid population in the local regulation model.

652 Curves gives the linear approximation for the fixation probability (Eq. (9a)). Parameters are the

653 same as in Fig. 3 and Fig. 8. The frequency of haploids is held fixed at (a)(b)(e)(f) $\hat{\rho}_{H}^{L}=0.8$,

$654(\mathrm{c})(\mathrm{d})(\mathrm{g})(\mathrm{h}) \hat{\rho}_{H}^{L}=0.3$. Fitness of haploids is higher than diploids in panels (a)(c)(e)(g)

$655\left(w_{H}>w_{D}\right)$, and the opposite condition is considered in panels $(\mathrm{b})(\mathrm{d})(\mathrm{f})(\mathrm{h})\left(w_{H}<w_{D}\right)$. Selection

656 only occurs in the haploid $(\mathrm{a})(\mathrm{b})(\mathrm{c})(\mathrm{d})$ or diploid stage $(\mathrm{e})(\mathrm{f})(\mathrm{g})(\mathrm{h})$.

657

\section{Supporting information}

659 S1. Supplementary Mathematica file. 
Figure1

(a)

Global regulation

Local regulation
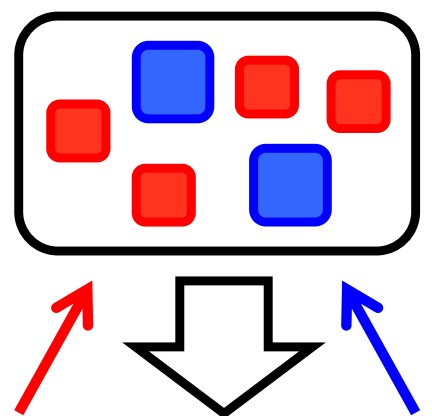

haploid

diploid
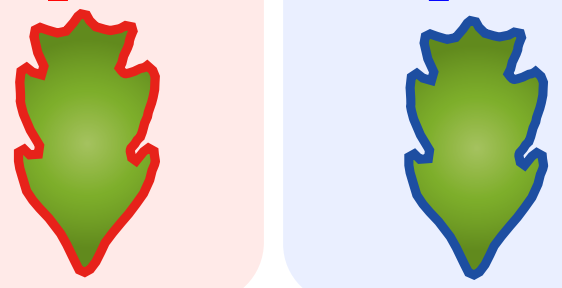

Total population size: $N_{\text {tot }}$
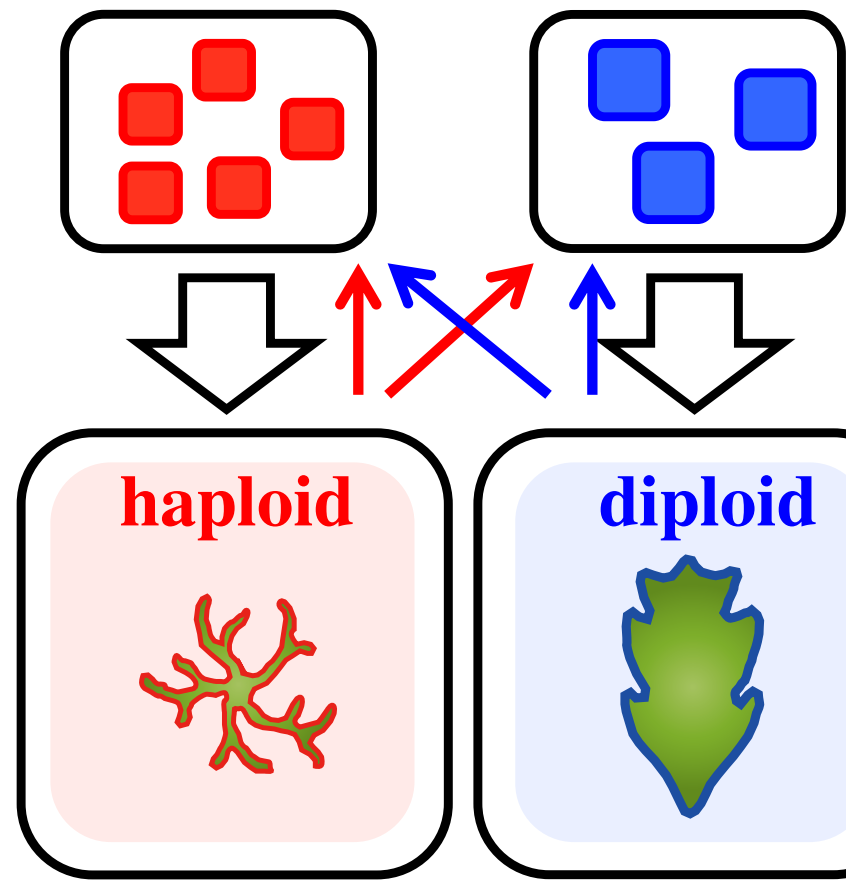

$N_{\mathrm{H}}$

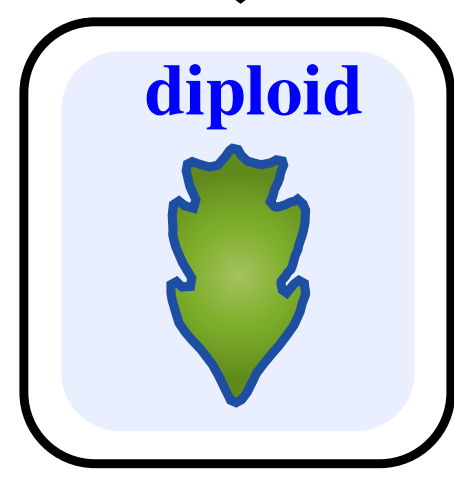

$N_{\mathrm{D}}$ 


\section{Figure 2}

(a)

$$
w_{H}>w_{D}
$$

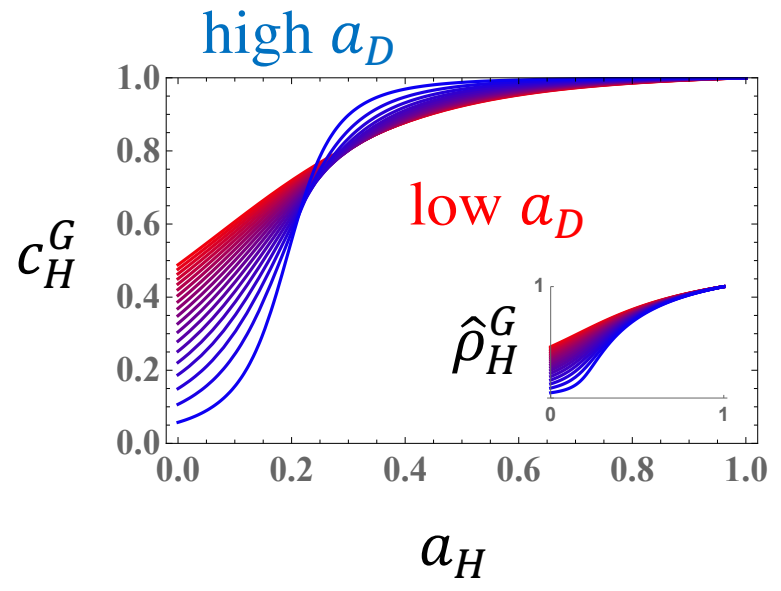

(b)

$$
w_{H}<w_{D}
$$

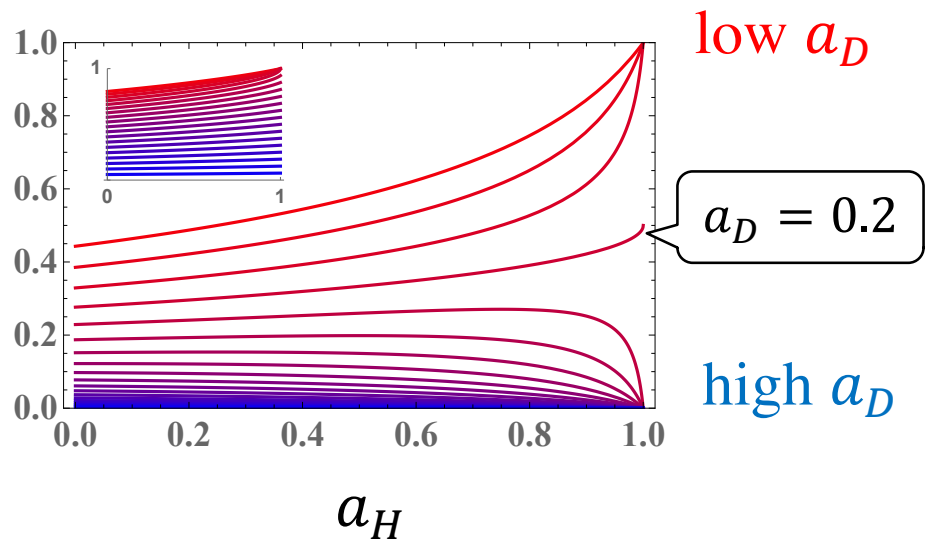




\section{Figure 3}

(a)

$$
w_{H}>w_{D}
$$

high $a_{D}$

$\hat{\rho}_{H}^{L}=0.8 \quad c_{H}^{L}$

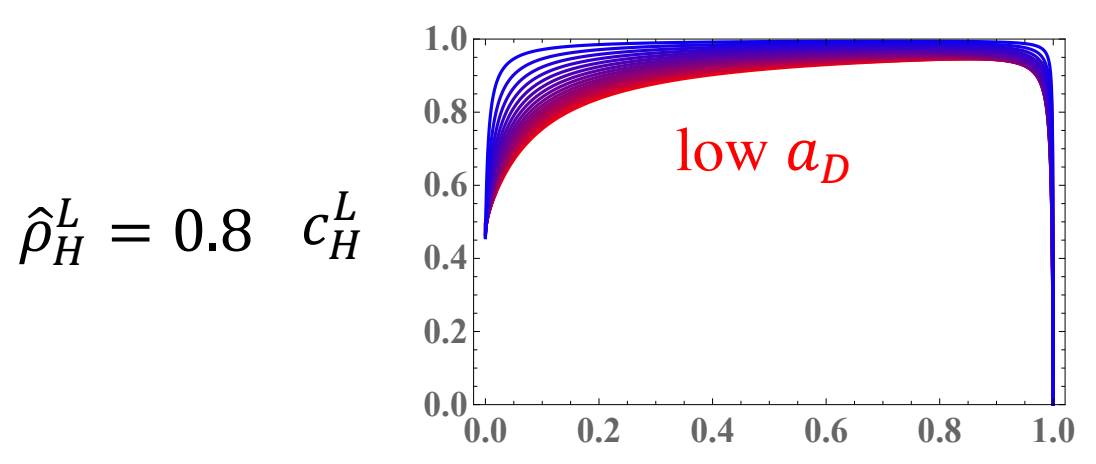

(c)

$\hat{\rho}_{H}^{L}=0.3 \quad c_{H}^{L}$

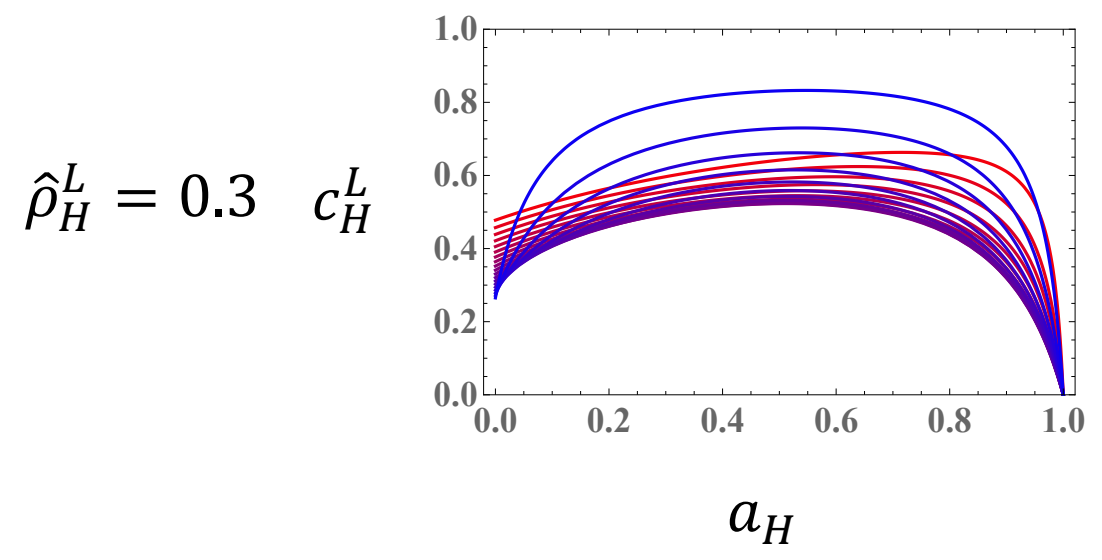

(b)

$w_{H}<w_{D}$

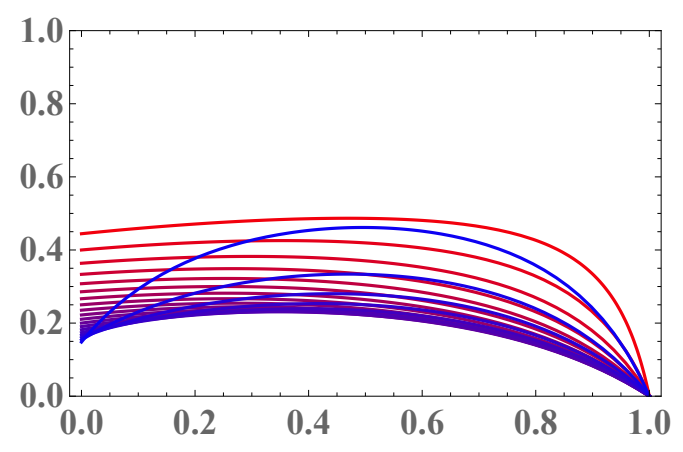

(d)

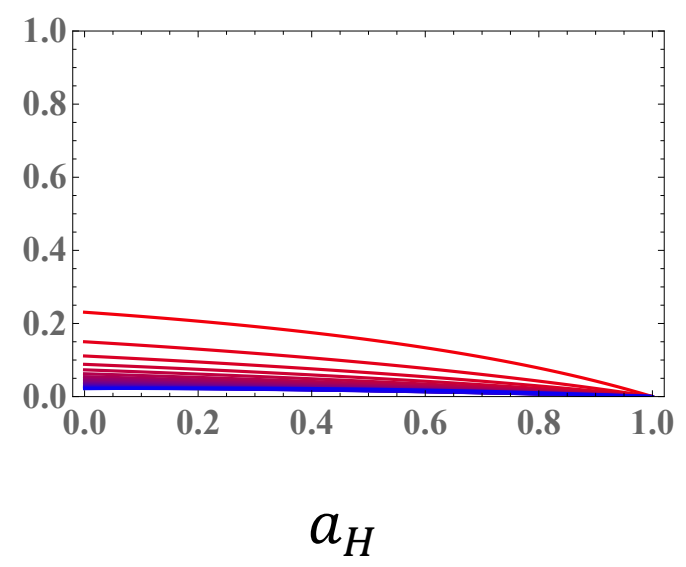




\section{Figure 4}

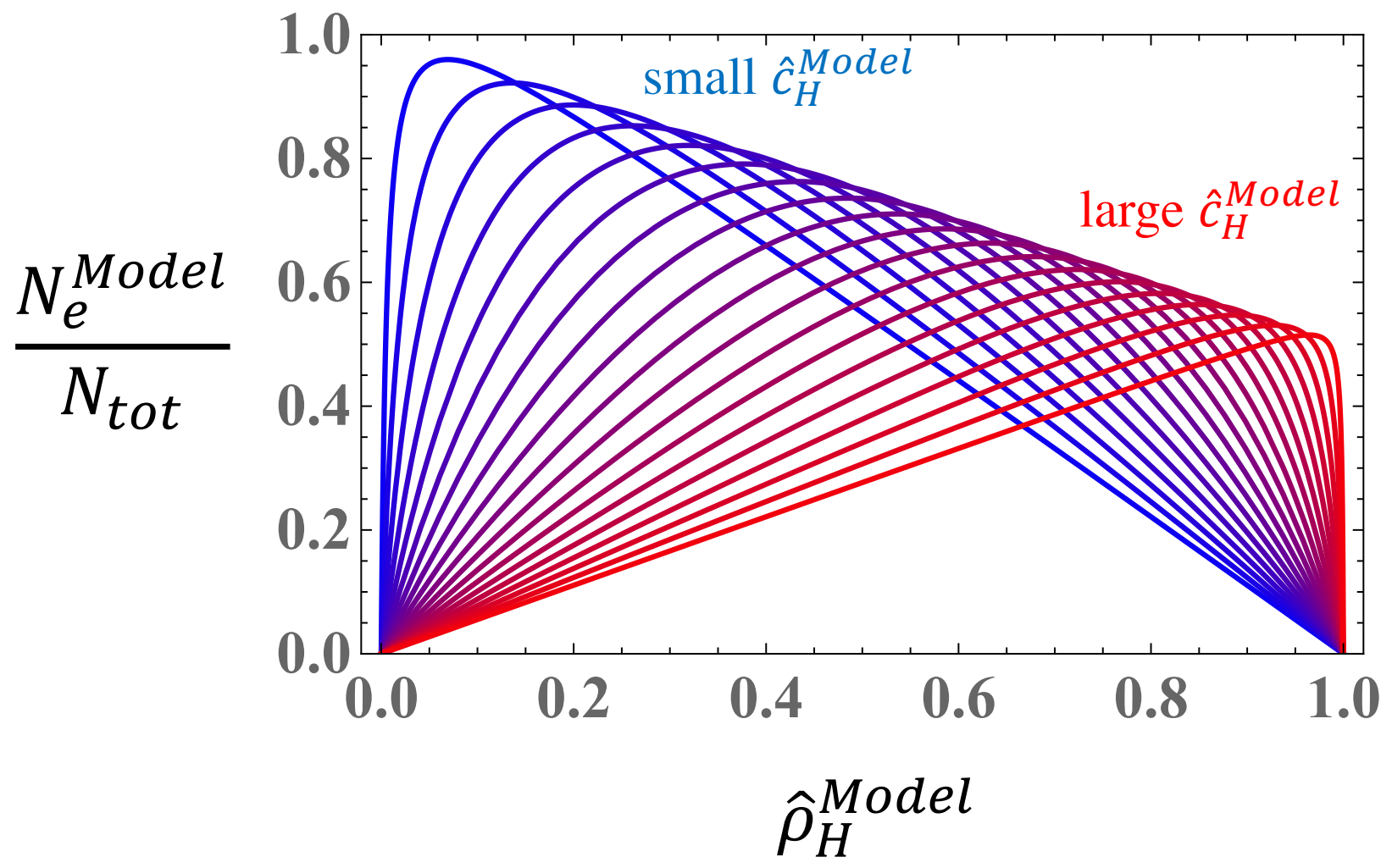




\section{Figure 5}

(a)

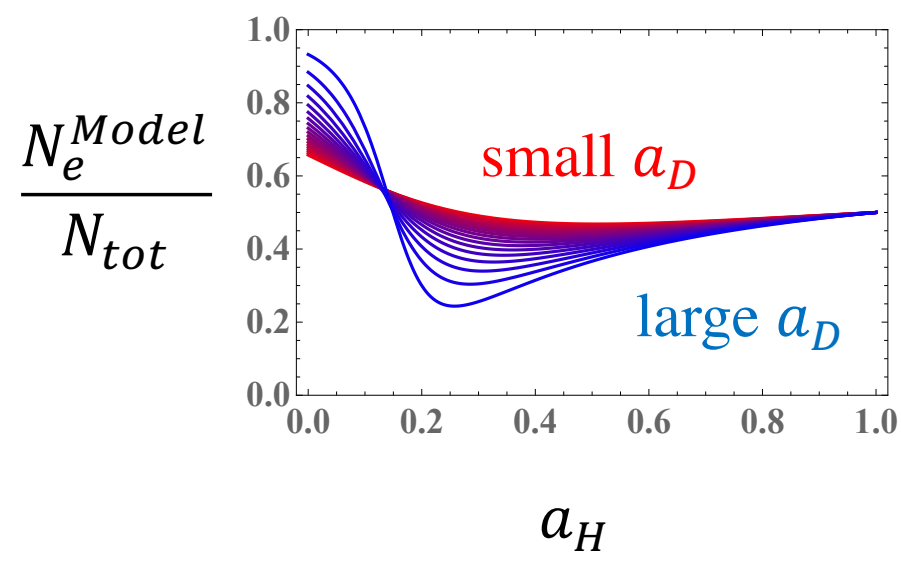

(b)

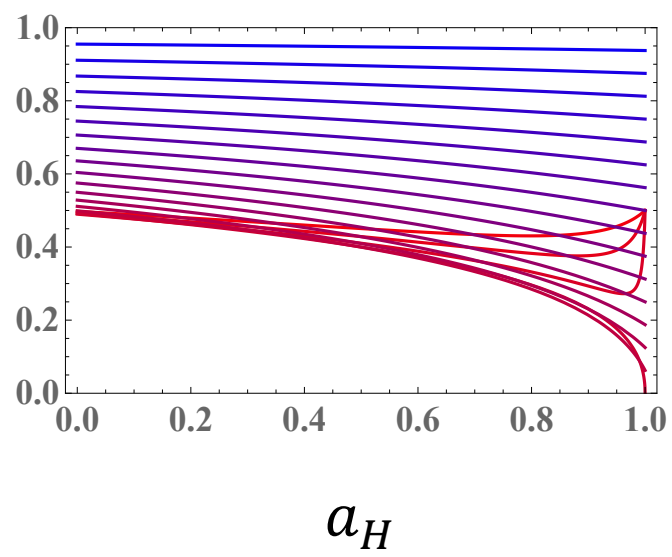




\section{Figure 6}

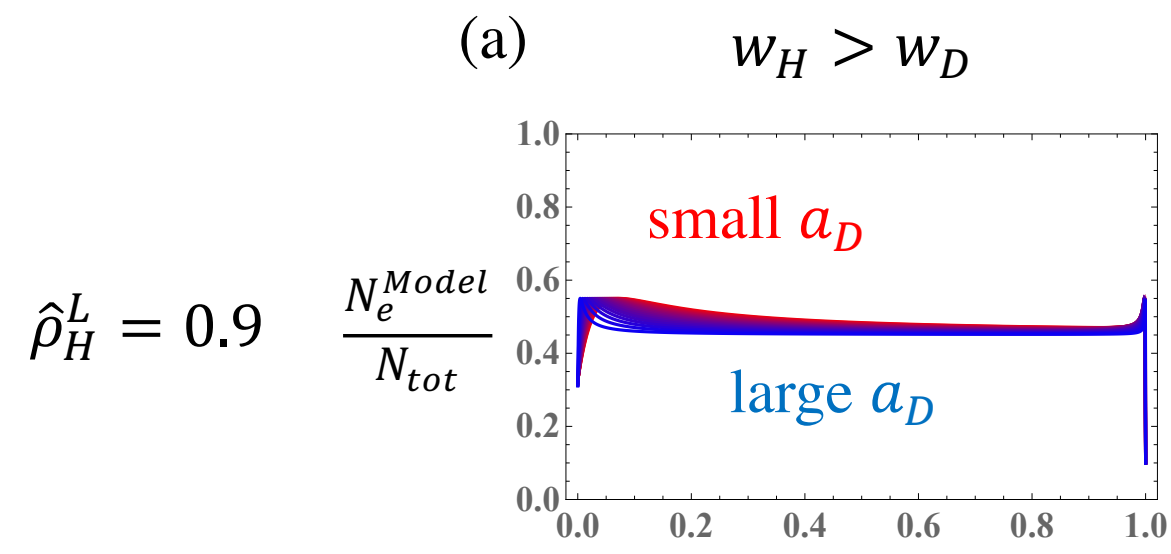
(b) $w_{H}<w_{D}$

(c)

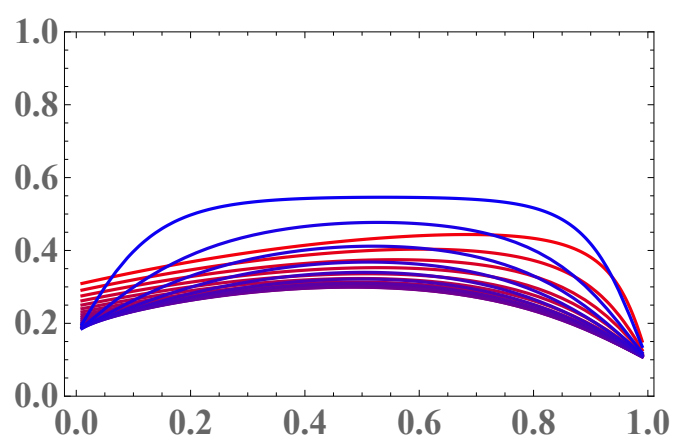

(d)
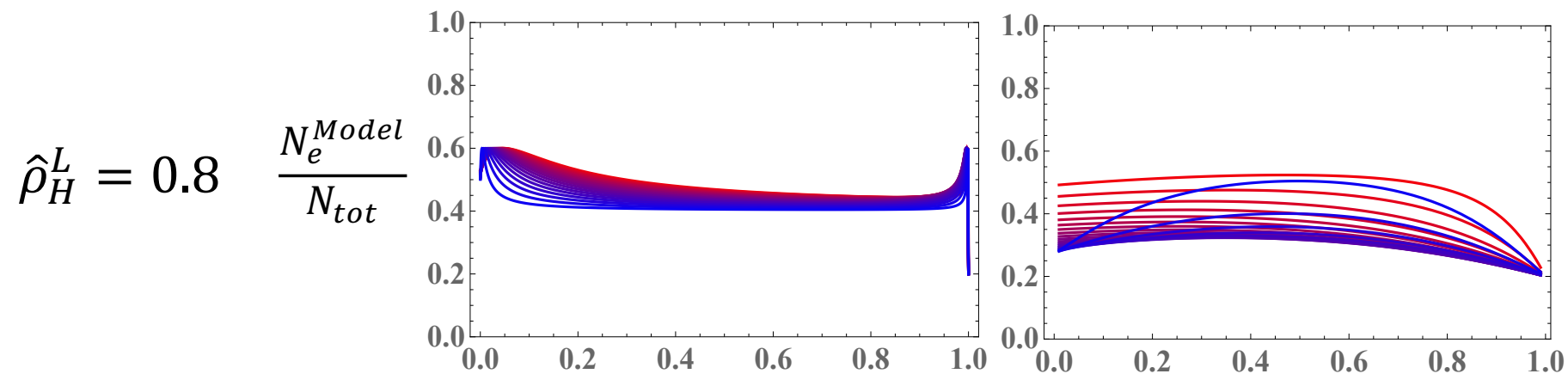

(e)

(f)

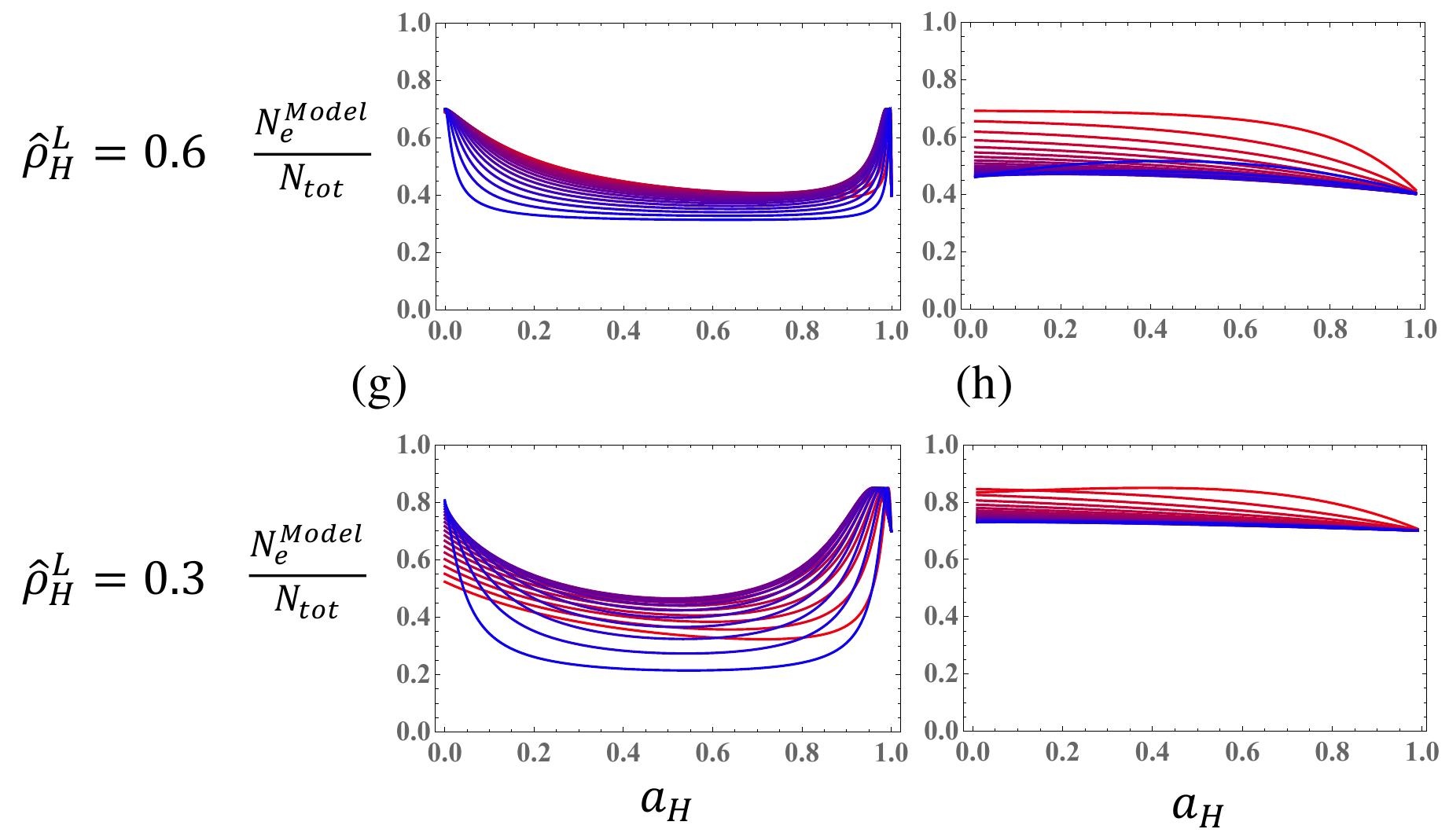




\section{Figure 7}

(a) global regulation

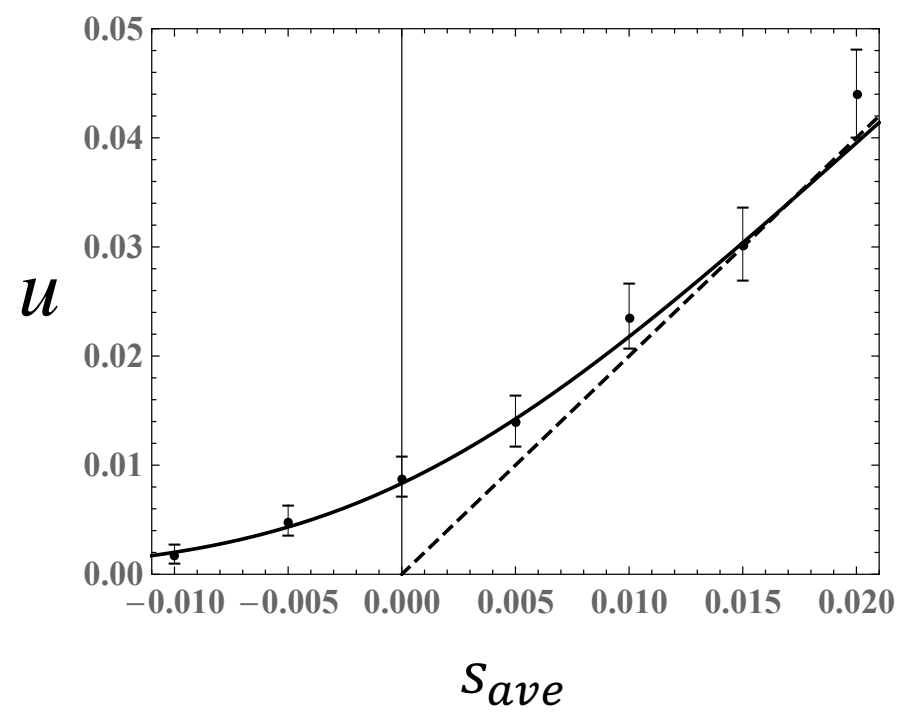

(b) local regulation

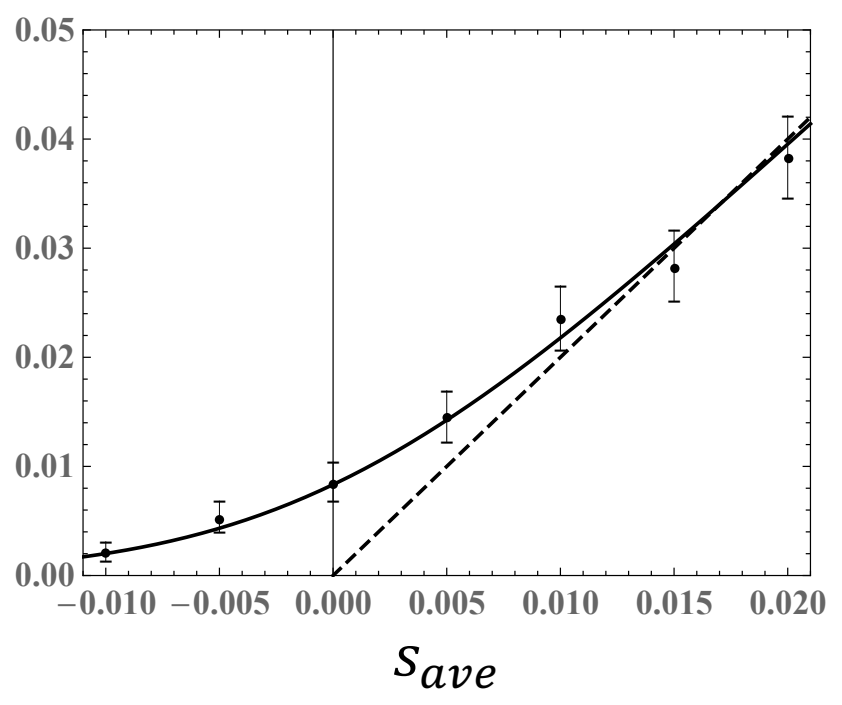


Figure 8

(a)

$$
w_{H}>w_{D}
$$

Haploid selection

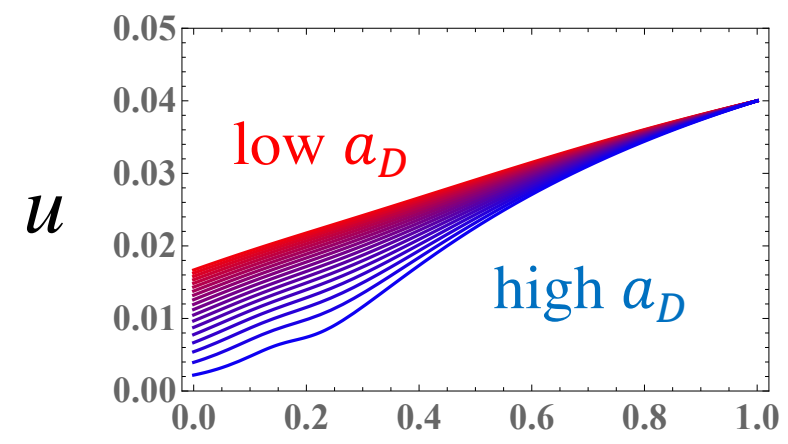

(c)

Diploid selection

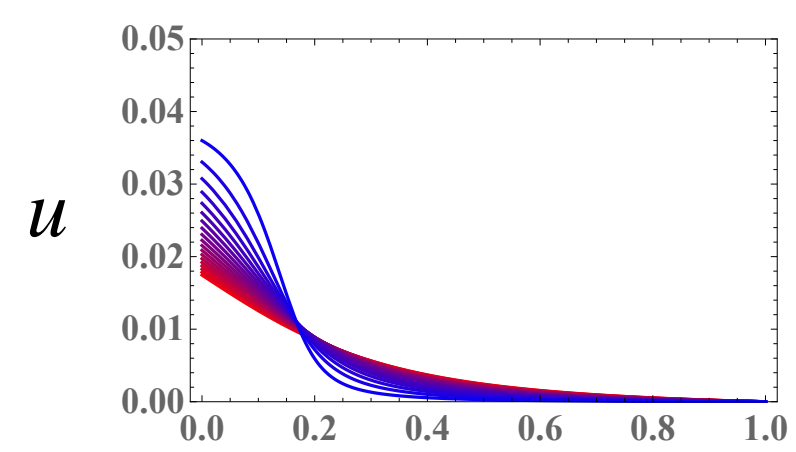

$a_{H}$ (b) $\quad w_{H}<w_{D}$

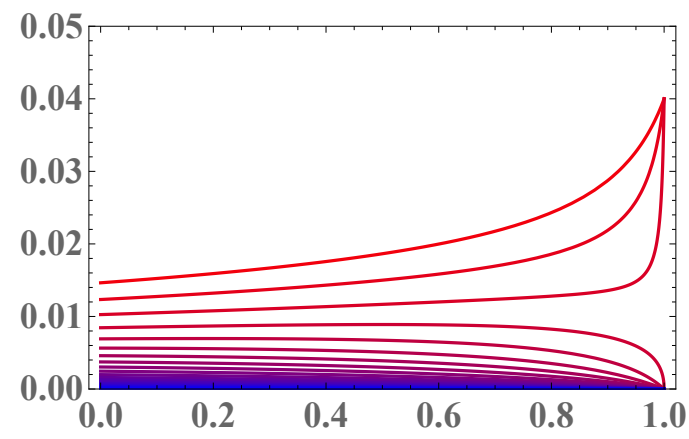

(d)

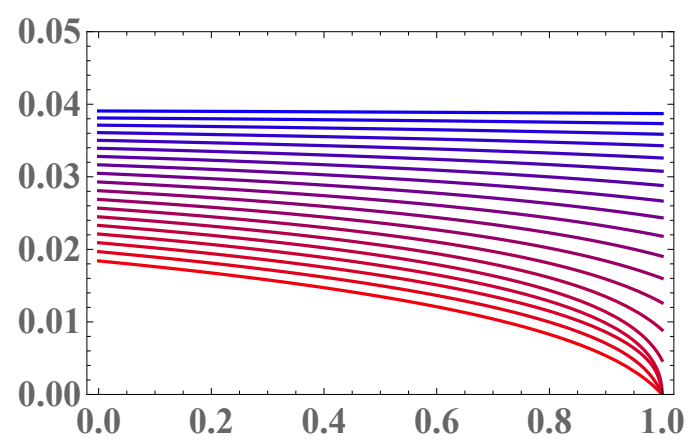

$a_{H}$ 
Figure 9

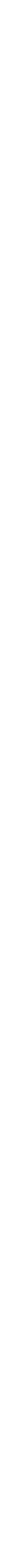

